\title{
AS ESPÉCIES DE EPHEMEROPTERA (INSECTA) REGISTRADAS PARA O BRASIL
}

\author{
Frederico Falcão Salles ${ }^{1,2}$; Elidiomar Ribeiro Da-Silva ${ }^{3}$ Michael D. Hubbard ${ }^{4}$ \& José Eduardo Serrão ${ }^{5}$
}

Biota Neotropica v4 (n2) - http://www.biotaneotropica.org.br/v4n2/pt/abstract?inventory+BN04004022004

Recebido em: 11/07/2004

Publicado em:27/10/2004

1. Museu de Entomologia, Departamento de Biologia Animal, Universidade Federal de Viçosa. Viçosa, Minas Gerais, Brasil. www.insecta.ufv.br/Entomologia/cien/sistematica/ephemeroptera/ephembrasil.htm

2. Autor correspondente. E-mail: ffsalles@insecta.ufv.br

3. Laboratório de Insetos Aquáticos (LABIAQUA), Departamento de Ciências Naturais, ECB, Universidade do Rio de Janeiro, CEP 20211-040, Rio de Janeiro, Rio de Janeiro, Brasil. www.unirio.br

4. Laboratory of Aquatic Entomology. Florida A\&M University, Tallahassee, Florida 32307, USA. www.famu.org/mayfly

5. Universidade Federal de Viçosa. Departamento de Biologia Geral, Viçosa, Minas Gerais, Brasil. www.ufv.br

\begin{abstract}
(The species of mayflies (Ephemeroptera: Insecta) recorded from Brazil). A checklist of the Brazilian Ephemeroptera fauna with all species, genera and families recorded from the country, including the states for which every species is reported, and the pertinent reference is presented. Comments on the status of knowledge of the Brazilian mayfly fauna are also provided. Up to date, 10 families, 63 genera, and 166 species are recorded. Baetidae and Leptophlebiidae have more than $50 \%$ of all records, while the North and Southeastern regions are significantly better studied than the other areas of the country.
\end{abstract}

Key words: Brazil, mayflies, records, status of knowledge, South-America.

\section{Resumo}

(As espécies de Ephemeroptera (Insecta) registradas para o Brasil). Uma lista da fauna de Ephemeroptera do Brasil, com todas as espécies, gêneros e famílias registrados para o país é apresentada, incluindo os estados para os quais as espécies estão reportadas assim como a bibliografia pertinente. Comentários acerca do estado atual do conhecimento da fauna brasileira também são tecidos. Até o presente momento 10 famílias, 63 gêneros e 166 espécies estão registrados. As famílias Baetidae e Leptophlebiidae compreendem mais de $50 \%$ de todos os registros, enquanto as regiões Norte e Sudeste são significativamente melhor estudadas que as demais.

Palavras-chave: Brasil, Ephemeroptera, registros, estado do conhecimento, América do Sul. 


\section{Introdução}

A ordem Ephemeroptera, composta atualmente por cerca de 4000 espécies, constitui o grupo mais antigo dentre os insetos alados. A incapacidade de dobrar suas asas sobre o abdome, a presença de dez segmentos abdominais, assim como a pouca redução numérica de suas nervuras alares, são algumas das características consideradas arcaicas que persistem na ordem (Elouard et al. 2003).

Seus integrantes são obrigatoriamente anfibióticos, com imaturos aquáticos e adultos terrestres. Enquanto as ninfas de Ephemeroptera exibem uma variedade de estratégias alimentares (podem ser filtradoras, raspadoras, fragmentadoras, coletoras ou até mesmo predadoras) e vivem de algumas semanas a poucos anos, os adultos não se alimentam, possuem as peças bucais atrofiadas e têm um curto período de vida, que em alguns casos não chega a mais de duas horas. Efemerópteros exibem ainda uma característica peculiar, presente apenas nessa ordem: a existência de um estágio alado intermediário entre a ninfa e o adulto, denominado subimago ou subadulto. Ao contrário dos adultos, encontrados com relativa freqüência revoando sobre ou nas proximidades dos corpos d'água, as subimagos são menos ativas, ficando em geral pousadas às margens dos ambientes dos quais emergiram.

As ninfas de Ephemeroptera constituem um dos principais grupos dentre os macroinvertebrados bentônicos. Além de serem extremamente abundantes e diversas, ocupam a maior parte dos meso-hábitats disponíveis, desde aqueles em áreas de remanso até os de forte correnteza. Como são em grande parte herbívoras ou detritívoras, e servem de alimento para uma série de predadores, como outros insetos e peixes, representam um importante elo na cadeia trófica dos ambientes aquáticos. Em função das distintas respostas apresentadas por suas espécies à degradação ambiental, os Ephemeroptera estão também entre os grupos mais utilizados em programas de biomonitoramento de qualidade da água.

O conhecimento a respeito dos Ephemeroptera no Brasil, apesar de ainda incipiente, aumentou consideravelmente nos últimos anos. Um significativo aumento no número de artigos publicados, tanto tratando da descrição de novos táxons (Ferreira \& Domínguez 1992, Da-Silva \& Pereira 1993, Lugo-Ortiz \& McCafferty 1995, 1996a, b, c, 1998, Molineri 1999, 2001, 2002, Salles \& LugoOrtiz 2002a, 2003a, b, Salles et al. 2003a, Lopes et al. 2003b, entre outros) como acrescentando novos registros de distribuição de espécies já conhecidas (Da-Silva 1992, 1997, 2003, Francischetti et al. 2003, Salles et al. 2003b, c, 2004b, aceito), praticamente dobrou, em duas décadas, o número de espécies reportado para o Brasil. No entanto, como o último catálogo publicado envolvendo a ordem no país data da década de 1980 (Hubbard 1982) e inexistem trabalhos abrangentes relacionados à fauna brasileira de Ephemeroptera, podemos considerar que as informações acerca da ordem ainda encontram-se bastante dispersas. A única exceção a isso diz respeito a duas listas, uma tratando das espécies registradas para o Brasil e outra das espécies registradas para o Estado de São Paulo, publicadas no site Ephemeroptera Galctica. No entanto, pode-se afirmar que ambas as listas encontram-se desatualizadas.

Informações pertinentes à distribuição das espécies no país, por exemplo, na maioria das vezes só podem ser acessadas através de trabalhos descritivos, muitas vezes antigos e nem sempre encontrados com facilidade. E, apesar de menos comum atualmente, gêneros e famílias ausentes até mesmo na América do Sul (e.g. Baetis Leach, 1815, Pseudocloeon Klapálek, 1905, Siphlonuriidae, Trichorythidae, entre outros) são eventualmente citados em artigos científicos brasileiros. Fato esse que também pode ser associado à ausência de chaves de identificação próprias para o país (Da-Silva et al. 2003).

No presente trabalho, tendo como objetivo principal integrar o conhecimento acerca da fauna brasileira de Ephemeroptera, apresentamos uma lista das espécies registradas para o Brasil, acompanhada de suas distribuições por estado e da bibliografia pertinente a cada registro. Em função desses dados, comentários a respeito do panorama atual relativo ao conhecimento da ordem no país também são tecidos. A lista originada a partir do presente trabalho está sendo disponibilizada on-line, no site Ephemeroptera do Brasil, com o intuito de mantê-la atualizada.

\section{Material e métodos}

A lista apresentada foi baseada primariamente no catálogo de Ephemeroptera da América do Sul (Hubbard 1982), acrescida dos artigos com referência ao país publicados desde então até julho de 2004. A partir do conhecimento referente às espécies de Ephemeroptera registradas para o Brasil, todos os artigos que tratavam da descrição dessas espécies, ou que as registravam para alguma localidade no país, foram revisados a fim de se obter os estados para os quais elas se encontravam reportadas.

A listagem, apresentada em ordem alfabética, encontra-se dividida em seções destinadas às famílias, subdivididas por sua vez em gêneros e finalmente em espécies. Autor e ano dos gêneros e espécies, assim como os sinônimos de cada espécie, quando existentes, são fornecidos, permitindo rápido acesso às descrições originais. Citações complementares às descrições originais também foram adicionadas à lista, de forma que o leitor possa ter acesso a toda bibliografia utilizada no trabalho.

Após o nome das espécies, a sigla do(s) estado(s) onde ela se encontra registrada aparece entre colchetes. Quando uma espécie está registrada para mais de um estado, os da mesma região encontram-se separados por vírgulas, enquanto aqueles de regiões distintas aparecem separados por ponto e vírgula. As siglas seguem o padrão normal para abreviação dos estados: Região Sul. PR, Paraná; RS, Rio 
Grande do Sul; SC, Santa Catarina. Região Sudeste. ES, Espírito Santo; MG, Minas Gerais; RJ, Rio de Janeiro; SP, São Paulo. Região Centro-Oeste. DF, Distrito Federal; GO, Goiás; MS, Mato Grosso do Sul; MT, Mato Grosso. Região Nordeste. BA, Bahia. Região Norte. AC, Acre; AM, Amazonas; PA, Pará; RO, Rondônia; RR, Roraima. Alguns estados das regiões Nordeste e Norte não constam na lista, uma vez que para os mesmos não foram encontrados registros de nenhuma espécie de Ephemeroptera (veja Discussão). Em poucos casos, ou por faltar uma referência mais específica sobre a localidade (alguns trabalhos antigos citavam a localidade da espécie apenas como Brasil), ou pela referência constar apenas de uma localidade sem especificação do estado (e várias localidades homônimas ocorrem em diferentes estados), é utilizada a seguinte simbologia [??]. E, finalmente, quando não se sabe o estado, porém a região onde a espécie foi encontrada é conhecida, o nome da mesma é escrito por extenso.

O presente trabalho não tem por finalidade discutir a validade das espécies descritas nem como dos registros fornecidos, seu objetivo é listar as espécies registradas para o Brasil que constam em artigos científicos. Entretanto, três espécies merecem alguns comentários: Palingenia atrostoma (Weber, 1801) (Palingeniidae), Ephoron umbratum (Hagen, 1888) (Polymitarcyidae) e Deleatidium vittatum Thew, 1960. Uma vez que a atual distribuição da família Palingeniidae e dos gêneros Ephoron Williamson, 1802 e Deleatidium Eaton, 1899 (Leptophlebiidae), não inclui a Região Neotropical, dificilmente essas espécies pertencem a esses gêneros, ou mesmo à Palingeniidae no caso de $P$. atrostoma. De acordo com Hubbard (1982), P. atrostoma trata-se provavelmente de uma Hexagenia (Ephemeridae) como abordado por Hagen (1871), enquanto E. umbratum seja possivelmente um Tortopus (Polymitarcyidae). D. vittatum, por sua vez, a despeito do que ocorreu com as demais espécies sul-americanas de Deleatidium, não foi transferida para Meridialaris Peters \& Edmunds, 1972. Contudo, de acordo com Peters \& Edmunds (1972), a espécie também não pertence a Deleatidium e só não foi transferida para outro gênero uma vez que até então só se conhecia a fêmea da espécie. As três espécies estão sendo tratadas como incertae sedis no presente trabalho. P. atrostoma e E. umbratum, por serem consideradas nomina dubia, não estão sendo tratadas durante a discussão.

\section{Resultados}

Tabela 1.

\section{Discussão}

Ao todo está registrado para o Brasil um total de dez famílias, 63 gêneros e 166 espécies de Ephemeroptera. Das famílias registradas para o país, Coryphoridae,
Melanemerellidae e Ephemeridae são representadas por apenas uma espécie (Figs 1 e 2). No caso das duas primeiras, ambas são monotípicas, sendo Melanemerellidae endêmica para o Brasil até o momento.

Dentre as famílias mais numerosas, Baetidae e Leptophlebiidae se destacam, comportando ao todo mais de $60 \%$ dos gêneros e $50 \%$ das espécies brasileiras. Esse grande número de espécies e gêneros pode ser devido: (i) à própria diversidade dos dois grupos, Baetidae e Leptophlebiidae compreendem uma grande porcentagem dos gêneros e espécies de Ephemeroptera em todo o mundo; e (ii) o fato de terem sido o alvo principal de grande parte dos trabalhos recentes lidando com a ordem na América do Sul. Das cerca de 70 espécies de Ephemeroptera registradas para o Brasil a partir da década de 1980, 45 pertencem a essas duas famílias.

As famílias Caenidae, Leptohyphidae e Polymitarcyidae, comportam basicamente a totalidade das espécies e gêneros restantes. Enquanto Caenidae e principalmente Leptohyphidae, desde as décadas de 1980 (Malzacher 1986, 1990, 1998, Pereira \& Da-Silva 1990a, DaSilva 1993a, b) e final da de 1990 (Molineri 1999, 2001, 2002, 2003), respectivamente, tiveram seu conhecimento significativamente incrementado. Com relação a Polymitarcyidae, trabalhos recentes a respeito do grupo são praticamente inexistentes, sendo a validade de muitas de suas espécies questionável.

Euthyplociidae, a despeito do reduzido número de táxons registrados para o Brasil, só não está representada no país por um gênero e duas de suas espécies até o momento descritas para todo o continente americano. Domínguez et al. (2002) sugerem que sua situação em toda a América do Sul não seja muito alterada, talvez com apenas uma ou outra espécie ainda estando por ser descrita.

Oligoneuriidae, por outro lado, encontra-se numa situação pouco usual. Apesar do baixo número de espécies reportadas para o Brasil, o de gêneros pode ser considerado relativamente elevado. Não só tal contraste aponta para um número alto de espécies por serem descritas, como também a diversidade de gêneros como Lachlania Hagen, 1868 e Homoeoneuria Eaton, 1881, em outras áreas do continente americano.

No que diz respeito ao conhecimento da ordem com relação às localidades brasileiras é facilmente notado que determinados estados e regiões concentram a grande maioria dos registros, enquanto outras áreas representam verdadeiras lacunas (Fig. 3, Tabela 2). As regiões Sudeste e Norte são, seguidas de perto pela Região Sul, as com o maior número de registros de espécies, gêneros e famílias (Fig. 3). Das famílias reportadas para o Brasil, apenas as possivelmente endêmicas, Melanemerellidae e Coryphoridae não estão representadas no Norte e no Sudeste, respectivamente. A Região Sul, ao contrário, apesar de 


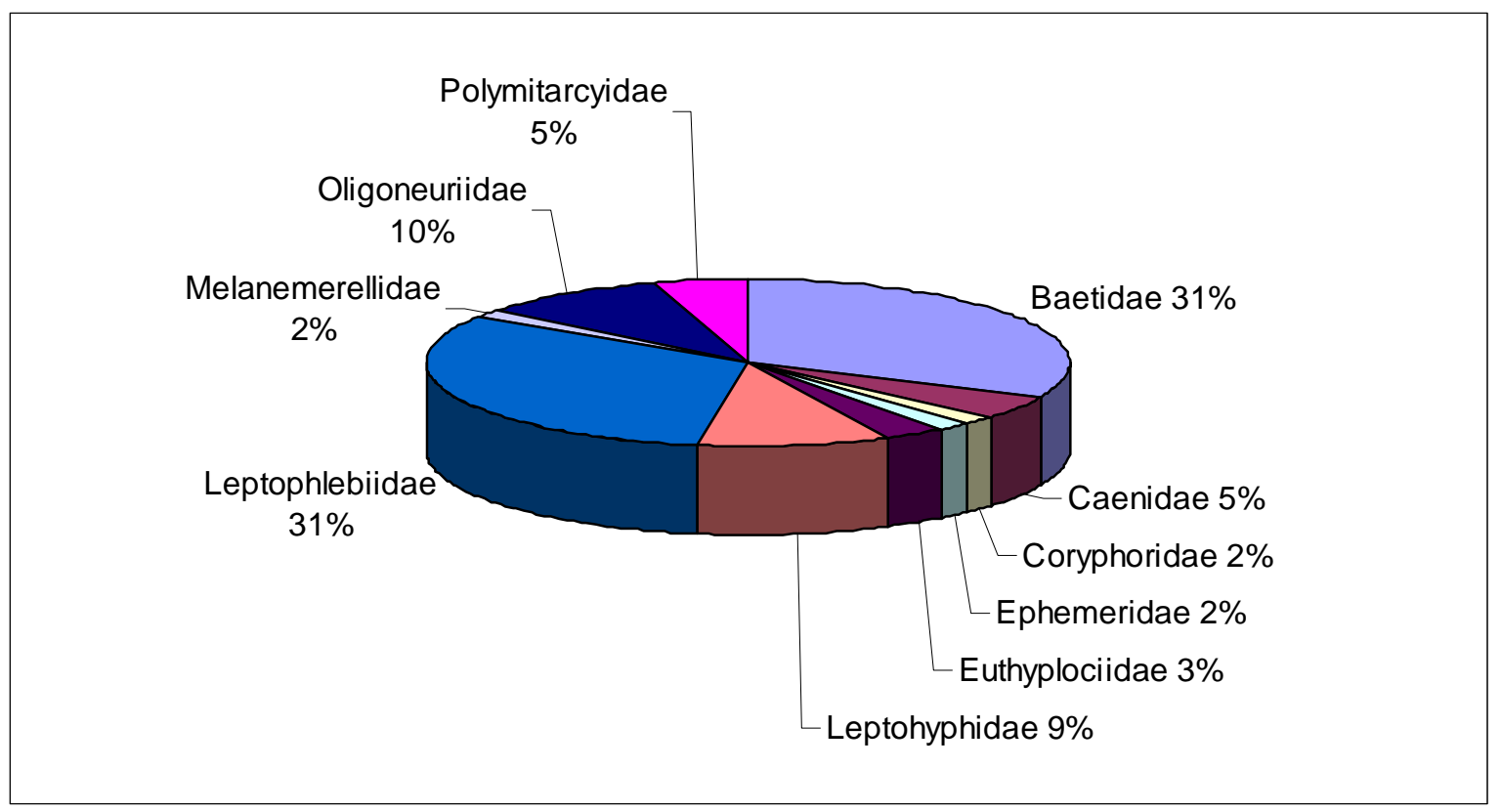

Figura 1. Proporção de gêneros de Ephemeroptera registrados para o Brasil com relação às famílias.

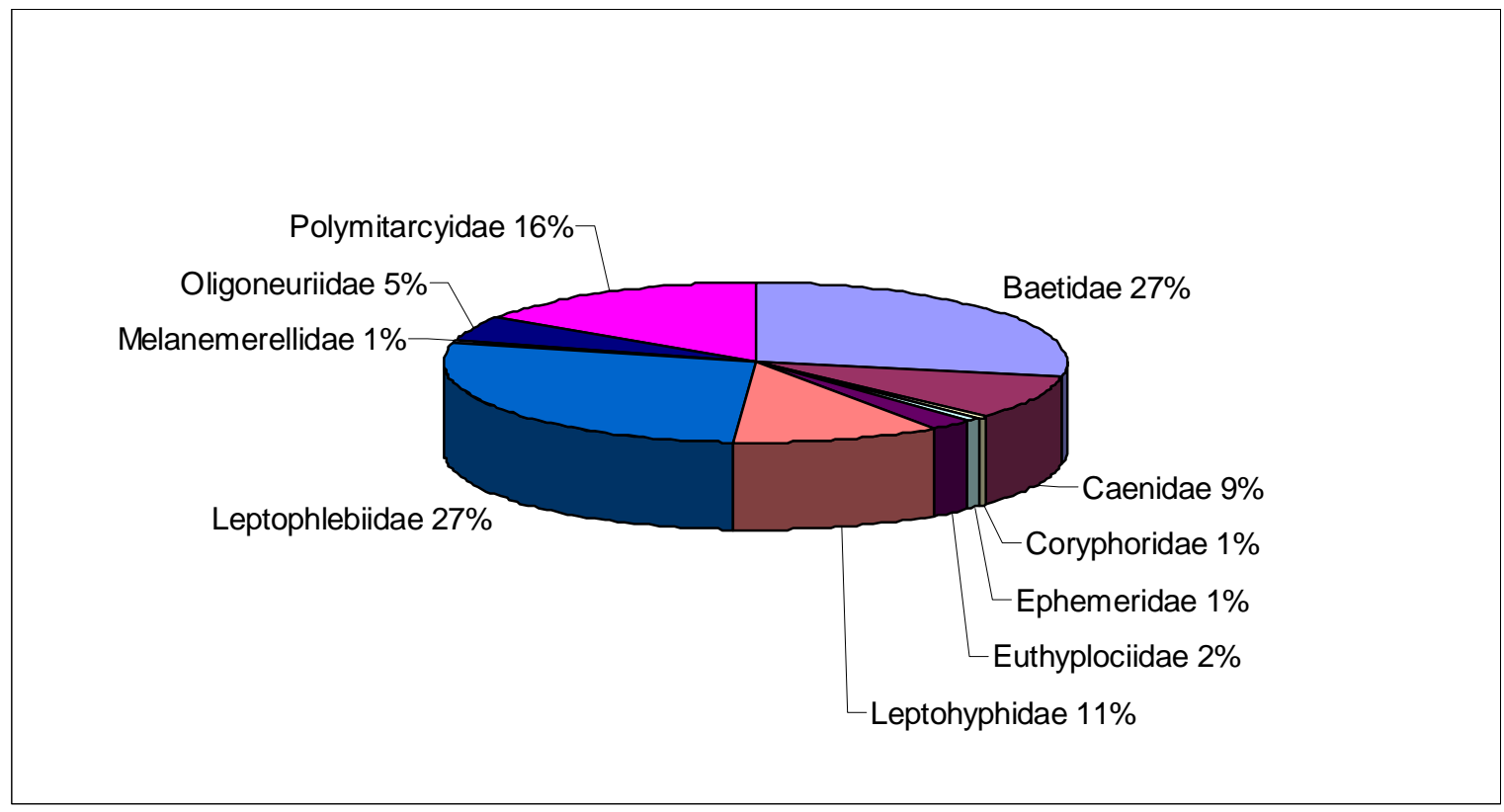

Figura 2. Proporção das espécies de Ephemeroptera registradas para o Brasil com relação às famílias.

http://www.biotaneotropica.org.br 


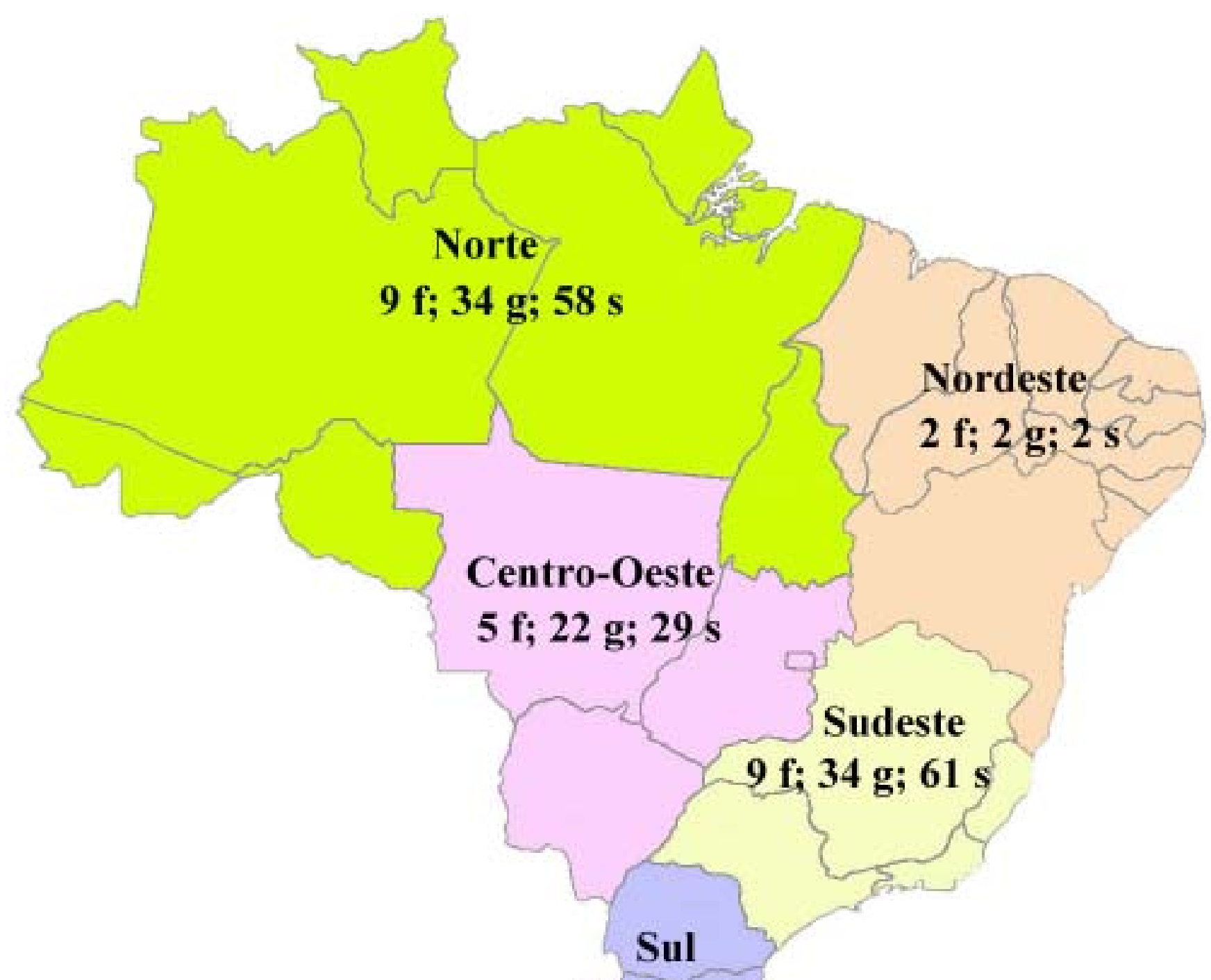

$6 \mathrm{f} ; 26 \mathrm{~g} ; 51 \mathrm{~s}$

Figura 3. Mapa do Brasil indicando respectivamente o número de famílias (f), gêneros (g) e espécies (s) de Ephemeroptera registrado para as suas cinco regiões. 
apresentar um número aproximado de espécies ao das duas regiões anteriores, tem um valor menor de gêneros e famílias.

Enquanto as regiões Sul e Sudeste apresentam um número semelhante de registros por estados (Tab. 2), exceto para o Espírito Santo, os dados da região Norte encontramse concentrados nos estados do Amazonas e Pará. Além disso, e tal situação só é similar no Nordeste (veja abaixo), alguns de seus estados como o Amapá e o Tocantins não possuem referência a nenhuma espécie nominal de Ephemeroptera. Da mesma forma, o que se conhece acerca da ordem no Acre, Rondônia e Roraima também pode ser considerado praticamente nulo.

Nas regiões Centro-Oeste e Nordeste, o conhecimento a respeito dos Ephemeroptera começa a ficar muito aquém do satisfatório. Áreas prioritárias para que se torne possível compreender a distribuição dos representantes da ordem no Brasil e, conseqüentemente, na América do Sul, são as menos conhecidas.

A região Centro-Oeste, segunda maior em extensão do Brasil ocupando quase $20 \%$ do território nacional, apresenta um número de registros, apesar de crescente recentemente, ainda restrito (Fig. 3). Não só diversas famílias não possuem espécies nominais relatadas para a região, como o conhecimento nos distintos estados e no Distrito Federal é fragmentado. Excetuando-se algumas pesquisas referentes à Baetidae desenvolvidas recentemente no Mato Grosso (Salles \& Batista 2004, Salles et al. no prelo), pouco tem sido acrescentado ao conhecimento das espécies de Ephemeroptera na região. Na região Nordeste, por sua vez, somente a Bahia, dos nove estados que a compõem, possui espécies formalmente reportadas. Além disso, tais registros estão restritos a apenas duas espécies, uma de Leptophlebiidae e outra de Oligoneuriidae.

O conhecimento a respeito dos Ephemeroptera no Brasil, apesar de típico para invertebrados em países tropicais, aumentou consideravelmente nas últimas duas décadas. Contudo, é evidente com base nos dados discutidos acima que esse conhecimento ainda é incipiente e assim permanecerá por algum tempo. A falta de especialistas no Brasil, a carência de chaves próprias para o país e/ou regiões, assim como a conseqüente escassez de trabalhos faunísticos envolvendo a ordem, podem ser considerados os principais fatores que colaboram para essa hipótese. Esperamos, portanto, que o presente trabalho seja uma ferramenta importante, incentivando e auxiliando futuros trabalhos relativos à fauna brasileira de Ephemeroptera.

\section{Agradecimentos}

A Marcela Miranda de Lima e Cesar Nascimento Francischetti, Universidade Federal de Viçosa, pelos inúmeros auxílios prestados durante a elaboração deste trabalho. Ao CNPq, por prover fundos a FFS como estudante do Programa de Pós-graduação em Entomologia na Universidade Federal de Viçosa e a ERDS como bolsista de produtividade em pesquisa (PQ).

\section{Referências bibliográficas}

ALLEN, R.K. 1967. New species of New World Leptohyphinae (Ephemeroptera: Tricorythidae). Can. Entomol. 99: 350-375.

ALLEN, R.K. 1973. New species of Leptohyphes Eaton (Ephemeroptera: Tricorythidae). Pan-Pac. Entomol. 49: 363-372.

ALLEN, R.K. \& MURVOSH, C.D. 1987. Mayflies (Ephemeroptera: Tricorythidae) of southwestern United States and northern Mexico. Ann. Entomol. Soc. Am. 80: $35-40$.

BANKS, N. 1913. The Stanford Expedition to Brazil. 1911. Neuropteroid insects from Brazil. Psyche 20: 83-89.

BERNER, L. \& THEW, T.B. 1961. Comments on the mayfly genus Campylocia with a description of a new species (Euthyplociidae: Euthyplociinae). Am. Midl. Nat. 66: 329336.

BURMEISTER, H. 1839. Handbuch der Entomologie, II. Band, 2. Ephemerina.

DA-SILVA, E.R. 1991. Descrição da ninfa de Callibaetis guttatus Navás, 1915, com notas biológicas e comentários sobre a imago (Ephemeroptera: Baetidae). An. Soc. Entomol. Bras. 20(2): 346-352.

DA-SILVA, E.R. 1992. Description of the nymph of Homoeoneuria (Notochora) fittkaui Pescador \& Peters, 1980 from northeastern Brazil (Ephemeroptera, Oligoneuriidae, Oligoneuriinae). Rev. Bras. Entomol. 36(3): 693-696.

DA-SILVA, E.R. 1993a. Descrição do imago macho de Caenis cuniana Froehlich, com notas biológicas (Ephemeroptera, Caenidae). Rev. Bras. Zool. 10(3): 413416.

DA-SILVA, E.R. 1993b. Efemerópteros da Serra dos Órgãos, Estado do Rio de Janeiro. II. Descrição de uma nova espécie de Leptohyphes Eaton, 1882 (Ephemeroptera, Tricorythidae). Rev. Bras. Entomol. 37(2): 313-316.

DA-SILVA, E.R. 1997. New and additional records of Leptophlebiidae (Ephemeroptera) from Rio de Janeiro State, Brazil. Rev. Biol. Trop. 44(3)/45(1): 684-685.

DA-SILVA, E.R. 2002a. Leptophlebiidae (Insecta: Ephemeroptera) ocorrentes no Estado do Rio de Janeiro: taxonomia e caracterização biológica das ninfas. Tese de doutorado. Rio de Janeiro, Universidade Federal do Rio de Janeiro, Museu Nacional. 
DA-SILVA, E.R. 2002b. Variações intraespecíficas da ninfa de Askola froehlichi Peters, 1969 (Insecta, Ephemeroptera, Leptophlebiidae), com notas biológicas. Bol. Mus. Nac., N.S. Zoo. 492: 1-5.

DA-SILVA, E.R. 2002c. Descrição da ninfa de Farrodes carioca Domínguez, Molineri \& Peters, 1996 (Insecta, Ephemeroptera, Leptophlebiidae). Bol. Mus. Nac., N.S. Zoo. 495: 1-5.

DA-SILVA, E.R. 2003. Ninfas de Thraulodes Ulmer, 1920 (Insecta: Ephemeroptera: Leptophlebiidae) ocorrentes no Estado do Rio de Janeiro, Brasil. Biota Neotrop. 3(2): 1-7.

DA-SILVA, E.R. \& LOPES, M.J.N. 2002. First record of Ulmeritoides missionensis (Ephemeroptera: Leptophlebiidae) in Brazil. Rev. Biol. Trop. 49(3/4): 12811282.

DA-SILVA, E.R. \& PEREIRA, S.M. 1992. Description of the nymph of Ulmeritus (U.) saopaulensis (Traver, 1947) from southeastern Brazil (Ephemeroptera, Leptophlebiidae, Atalophlebiinae). Rev. Bras. Entomol. 36(4): 855-858.

DA-SILVA, E.R. \& PEREIRA, S.M. 1993. Efemerópteros da Serra dos Órgãos, Estado do Rio de Janeiro. III. Descrição de uma nova espécie de Lachlania Hagen, 1868 (Ephemeroptera: Oligoneuriidae). An. Acad. Bras. Cienc. 65(3): 296-301.

DA-SILVA, E.R. SALLES, F.F., NESSIMIAN, J.L. \& COELHO, L.B.N. 2003. A identificação das famílias de Ephemeroptera (Insecta) ocorrentes no Estado do Rio de Janeiro: chave pictórica para as ninfas. Bol. Mus. Nac., N.S. Zoo. 508: 1-6.

DAY, W.C. 1955. New genera of mayflies from California (Ephemeroptera). Pan-Pac. Entomol. 31: 121-137.

DEMOULIN, G. 1955. Une mission biologique belge au Brésil. Éphéméroptères. Bull. Inst. R. Sci. Nat. Belg. 31(20): 132.

DEMOULIN, G. 1966. Contribution à l'étude des Ephéméroptères du Surinam. Bull. Inst. R. Sci. Nat. Belg. 42(37): 1-22.

DOMÍNGUEZ, E. 1995. Cladistic analysis of the UlmeritusUlmeritoides group (Ephemeroptera, Leptophlebiidae), with descriptions of five new species of Ulmeritoides. J. New York Entomol. Soc. 103: 15-38.

DOMÍNGUEZ, E. \& FLOWERS, R.W. 1989. A revision of Hermanella and related genera (Ephemeroptera: Leptophlebiidae: Atalophlebiinae) from Subtropical South America. Ann. Entomol. Soc. Amer. 82: 555-573.

DOMÍNGUEZ, E., MOLINERI, C. \& PETERS, W.L. 1996. Ephemeroptera from Central and South America: New species of the Farrodes bimaculatus group with a key for the males. Stud. Neotrop. Fauna Environ. 31: 87-101.
DOMÍNGUEZ, E., PETERS, W.L., PETERS, J.G \& SAVAGE, H.M. 1997. The image of Simothraulopsis Demoulin with a redescription of the nymph (Ephemeroptera: Leptophlebiidae: Atalophlebiinae). Aquatic Insects. 19(3): 141-150.

DOMÍNGUEZ, E., ZÚÑIGA, M.C. \& MOLINERI, C. 2002. Estado actual del conocimiento y distribución del orden Ephemeroptera (Insecta) en la Región Amazónica. Caldasia 24(2): 459-469.

DOMINIQUE, Y., THOMAS, A., ORTH, K. \& DAUTA, C. 2000. Les Ephémèrs de La Guyane Française. 2. Camelobaetidius billi et C. janae n. spp (Ephemeroptera, Baetidae). Ephemera 2: 39-48.

EATON, A.E. 1871. A monograph on the Ephemeridae. Trans. Entomol. Soc. London. 1871: 1-164.

EATON, A.E. 1881. An announcement of new genera of the Ephemeridae. Entomol. Mon. Mag. 17: 191-197.

EATON, A.E. 1883-1888. A revisional monograph of recent Ephemeridae or mayflies. Trans. Linn. Soc. London. 3: 1352.

EATON, A.E. 1899. An annotated list of the Ephemeridae of New Zealand. Trans. Entomol. Soc. London. 1899: 285293.

EDMUNDS JR, G.F. 1948. A new genus of mayflies from western North America (Leptophlebiinae). Proc. Biol. Soc. Washington. 61: 141-146.

EDMUNDS JR, G.F. 1950. Notes on neotropical Ephemeroptera. I. New and little known Leptophlebiidae. Rev. Entomol. 21(3): 551-554.

EDMUNDS JR, G.F. 1963. A new genus and species of mayfly from Peru (Ephemeroptera: Leptophlebiidae). PanPac. Entomol. 39: 34-36.

EDMUNDS JR, G.F., JENSEN, S.L. \& BERNER, L. 1976. The mayflies of North and Central America. Minneapolis, University of Minnesota Press.

ELOUARD, J.M., GATTOLLIAT, J.L. \& SARTORI, M. 2003. Ephemeroptera, mayflies. In The Natural History of Madagascar (Goodman S.M \& J.P. Benstead, eds). University of Chicago Press, Chicago, p.639-645.

ESBEN-PETERSEN, T. 1912. New and little-known species of Ephemerida from Argentine. (Neuropt.). Deutsch. Entomol. Zeitschr.: 333-342.

FERREIRA, M.J.N. \& DOMÍNGUEZ, E. 1992. Anew species of Hermanella (Ephemeroptera: Leptophlebiidae: Atalophlebiinae) from southeastern Brazil. Aquatic Insects. 14(3): 179-182.

FRANCISCHETTI, C.N., SALLES, F.F., LUGO-ORTIZ, C.R. \& DA-SILVA, E.R. 2003. First report of Americabaetis Kluge (Ephemeroptera: Baetidae) from Rio de Janeiro, Brazil. Entomotrópica. 18: 69-71. 
FROEHLICH, C.G. 1969. Caenis cuniana sp. n., a parthenogenetic mayfly. Beitr. Neotrop. Fauna. 6: 103-108.

GUERIN, E. \& PERCHERON, A.R. 1838. Genera des Insects. Livr. VI.

HAGEN, H. 1861. Synopsis of the Neuroptera of North America with a list of the South American species. Smithsonian Misc. Coll.: 1-347.

HAGEN, H. 1888. Unsere gegenwärtige Kenntniss der Ephemeren. Stett. Entomol. Z. 49: 221-232.

HOFFMAN, C., SARTORI, M. \& THOMAS, A. 1999. Les Ephéméroptères (Ephemeroptera) de la Guadeloupe (petites Antilles Françaises). Mem. Soc. Vaud. Sc. Nat. 20(1): 1-96.

HUBBARD, M.D. 1982. Catálogo abreviado de Ephemeroptera da América do Sul. Papéis Avulsos Zool. 34: 257-282.

LESTAGE, J.A. 1923. L'imbrglio campsurien. Notes critiques sur les Campsurus. Ann. Soc. Entomol. Belg.: 113-124.

LESTAGE, J.A. 1924. Atalophlebia bieni sp. n. Éphemère nouvelle du Brésil. Ann. Soc. Entomol. Belg. 64: 21-24.

LESTAGE, J.A. 1930. Notes sur le genre Massartella nov. gen. de la famille des Leptophlebiidae (Ephemeroptera) et le génotype Massartella brieni Lest. Une Mission Biologique Belge au Brésil 2: 249-258.

LOPES, M.J.N. 1999. Sistemática de Atalophlebiinae (Insecta: Ephemeroptera, Leptophlebiidae) nos escudos das Guianas e Brasileiro (Rondônia). Tese de doutorado. Manaus, Instituto Nacional de Pesquisas da Amazônia/ Universidade do Amazonas.

LOPES, M.J.N., DA-SILVA, E.R. \& PY-DANIEL, V. 2003b. A new species of Ulmeritoides from Brazil (Ephemeroptera : Leptophlebiidae). Rev. Biol. Trop. 51(1): 195-199.

LOPES, M.J.N., FROEHLICH, C.G \& DOMINGUEZ, E. 2003a. Description of the larva of Thraulodes schlingeri (Ephemeroptera, Leptophlebiidae). Iheringia S. Zool. 93(2): 197-200.

LUGO-ORTIZ, C.R. \& McCAFFERTY, W.P. 1995. Three distinctive new genera of Baetidae (Insecta, Ephemeroptera) from South America. Ann. Limnol. 31: 233-243.

LUGO-ORTIZ, C.R. \& McCAFFERTY, W.P. 1996a. Aturbina georgei gen et sp-n. A small minnow mayfly (Ephemeroptera, Baetidae) without turbinate eyes. Aquatic Insects. 18: 175-183.

LUGO-ORTIZ, C.R. \& McCAFFERTY, W.P. 1996b. The genus Paracloeodes (Insecta, Ephemeroptera, Baetidae) and its presence in South America. Ann. Limnol. 32: 161169.

LUGO-ORTIZ, C.R. \& McCAFFERTY,W.P. 1996c. Taxonomy of the Neotropical genus Americabaetis, new status (Insecta: Ephemeroptera: Baetidae). Stud. Neotrop. Fauna Environ. 31: 156-169.
LUGO-ORTIZ, C.R. \& McCAFFERTY, W.P. 1997. First report and new species of the genus Apobaetis (Ephemeroptera: Baetidae) from South America. Aquatic Insects. 19: 243-246.

LUGO-ORTIZ, C.R. \& McCAFFERTY, W.P. 1998. Five new genera of Baetidae (Insecta : Ephemeroptera) from South America. Ann. Limnol. 34: 57-73.

LUGO-ORTIZ, C.R., SALLES, F.F. \& FURIERI, K.S. 2002. First records of small minnow mayflies (Ephemeroptera; Baetidae) from the State of Espírito Santo, southeastern Brazil. Lundiana 3: 79-80.

MALZACHER, P. 1986. Caenidae aus dem Amazonasgebiet (Insecta, Ephemeroptera). Spixiana 9: 83-103.

MALZACHER, P. 1990. Neue Arten der EintagsfliegenFamilie Caenidae (Insecta, Ephemeroptera) aus Südamerika. Stud. Neotrop. Fauna Environ. 25(1): 31-39.

MALZACHER, P. 1998. Remarks on the genus Brasilocaenis (Ephemeroptera: Caenidae), with the description of a new species: Brasilocaenis mendesi. Stuttgarter Beitr. Naturk.

MAYO, V.N. 1968. Two new species of the genus Baetodes from Ecuador (Ephemeroptera: Baetidae). Pan-Pac. Entomol. 44: 251-257.

McCAFFERTY, W.P. 1970. Neotropical nymphs of the genus Hexagenia (Ephemeroptera: Ephemeridae). J. Georgia Entomol. Soc. 5: 224-228.

McCAFFERTY, W.P. \& LUGO-ORTIZ, C.R. 1995. Cloeodes hydation, n. sp. (Ephemeroptera: Baetidae) an extraordinary, drought tolerant mayfly from Brazil. Entomol. News. 106: 29-35.

MELO, S.M., TAKEDA, A.M. \& MONKOLSKI, A. 2002. Seasonal dynamics of Callibaetis willineri (Ephemeroptera, Baetidae) associated with Eichhornia azurea (Pontedericeae) in Guaraná Lake of the Upper Paran'a River, Brazil. Hydrobiol. 470: 57-62.

MOL, A.W.M. 1986. Harpagobaetis gulosus gen. nov., spec. nov., a new mayfly from Suriname (Ephemeroptera: Baetidae). Zool. Meded. 60: 63-70.

MOLINERI, C. 1999. Revision of the genus Tricorythopsis (Ephemeroptera: Leptohyphidae) with description of four new species. Aquatic Insects. 21: 285-300.

MOLINERI, C. 2001. Traverhyphes: a new genus of Leptohyphidae for Leptohyphes indicator and related species (Insecta: Ephemeroptera). Spixiana 24(2): 129140.

MOLINERI, C. 2002. Cladistic analysis of the South-American species of Trichorythodes (Ephemeroptera: Leptohyphidae) with the descriptions of new species and stages. Aquatic Insects. 24(4): 273-308.

MOLINERI, C. 2003. Revision of the South-American species of Leptohyphes Eaton (Ephemeroptera: Leptohyphidae) with a key to the nymphs. Stud. Neotrop. Fauna Environ. 38(1): 47-70. 
MOLINERI, C. \& DOMINGUEZ, E. 2003. Nymph and egg of Melanemerella brasiliana (Ephemeroptera : Ephemerelloidea : Melanemerellidae), with comments on its systematic position and the higher classification of Ephemerelloidea. J. N. Am. Benthol. Soc. 22(2): 263-275.

NAVÁS, L. 1912. Neurópteros nuevos de América. Broteria 10: 194-202.

NAVÁS, L. 1915a. Neurópteros nuevos o poco conocidos (Sexta Serie). Mem. R. Acad. Cienc. Artes Barcelona. 12: 119-136.

NAVÁS, L. 1915b. Neurópteros sudamericanos, segunda série. Broteria 13: 5-13.

NAVÁS, L. 1916. Neuroptera nova americana. Mem. Pontif. Accad. Rom. Nuovi Lincei. 2(2): 60-80.

NAVÁS, L. 1917. Algunos insectos Neurópteros de la Argentina. Physis 3: 186-196.

NAVÁS, L. 1918. Insectos chilenos. Bol. Soc. Aragonesa Cienc. Nat. 17: 212-230.

NAVÁS, L. 1919. Algunos insectos Neurópteros de la Argentina. Serie 2. Physis 9: 80-90.

NAVÁS, L., 1920a. Algunos insectos de Santa Fe (República Argentina) recogidos por el P. Juan C. Muhn, S. J. Estudios. 18: 131-135.

NAVÁS, L. 1920b. Insectos Sudamericanos. An. Soc. Cient. Argent. 90: 33-72.

NAVÁS, L. 1920c. Algunos insectos del Brasil. $3^{\text {a }}$ Serie. Rev. Mus. Paulista. 12: 413-417.

NAVÁS, L. 1922. Efemerópteros nuevos o poco conocidos. Bol. Soc. Entomol. España. 5: 54-63.

NAVÁS, L. 1923. Insecta nova. VIII Serie. Mem. Pontif. Accad. Rom. Nuovi Lincei. 6(2): 1-27.

NAVÁS, L. 1924. Insectos de la Argentina y Chile. Estudios: 358-368.

NAVÁS, L. 1929. Insectos de la Argentina. Quinta Serie. Rev. Soc. Entomol. Argent. 32: 219-225.

NAVÁS, L. 1930a. Insectos de la Argentina. Sexta Serie. Rev. Soc. Entomol. Argent. 3: 125-132.

NAVÁS, L. 1930b. Insectos neotropicos. $6^{a}$ Serie. Rev. Chil. Hist. Nat. 34: 62-75.

NAVÁS, L. 1931. Insectos del Brasil. 4a Serie. Rev. Mus. Paulista. 17: 455-458.

NAVÁS, L. 1932. Insectos de la Argentina. Rev. Acad. Cienc. Zaragoza. 16: 87-120.

NAVÁS, L. 1934. Insectos suramericanos. Octava Serie. Rev. Acad. Cient. Madrid. 31: 9-28.

NAVÁS, L. 1936. Insectos del Brasil. 5a Serie. Rev. Mus. Paulista. 20: 731-734.

NEEDHAM, J.G. \& MURPHY, H.E. 1924. Neotropical mayflies. Bull. Lloyd Lib. Bot. Pharm. Med., 24. Entomol. Ser. 4: 1-79.
NOLTE, U., OLIVEIRA, M.J. \& STUR, E. 1997. Seasonal, discharge-driven patterns of mayfly assamblages in an intermittent Neotropical stream. Freshwater Biol. 37: 333343.

PEREIRA, S.M. 1987. Presença de Lachlania Hagen, 1868 no Brasil: Descrição de uma nova espécie e notas sobre as demais (Ephemeroptera, Oligoneuriidae). Bol. Mus. Nac., N.S. Zoo. 314: 1-11.

PEREIRA, S.M. \& DA-SILVA, E.R. 1990a. Nova espécie de Caenis Stephens, 1835 do sudeste do Brasil (Ephemeroptera, Caenidae). Bol. Mus. Nac., N.S. Zoo. 341: 1-8.

PEREIRA, S.M. \& DA-SILVA, E.R. 1990b. Nova espécie de Campylocia Needham \& Murphy, 1924 com notas biológicas (Ephemeroptera, Euthyplociidae). Bol. Mus. Nac., N.S. Zoo. 336: 1-12.

PEREIRA, S.M. \& DA-SILVA, E.R. 1991. Descrição de uma nova espécie de Campsurus Eaton, 1868 do sudeste do Brasil, com notas biológicas (Ephemeroptera: Polymitarcyidae: Campsurinae). Rev. Bras. Biol. 51(2): 321-326.

PESCADOR, M.L. \& EDMUNDS JR, G.F. 1994. New genus of Oligoneuriidae (Ephemeroptera) from South America. Ann. Entomol. Soc. Amer. 87: 263-269.

PESCADOR, M.L. \& PETERS, W.L. 1980. A revision of the genus Homoeoneuria (Ephemeroptera: Oligoneuriidae). Trans. Amer. Entomol. Soc. 106: 357-393.

PESCADOR, M.L. \& PETERS, W.L. 1990. Biosystematics of the genus Massartella Lestage (Ephemeroptera: Leptophlebiidae: Atalophlebiinae) from South America. Aquatic Insects. 12: 145-160.

PETERS, W.L. 1969. Askola froehlichi, a new genus and species from southern Brazil (Leptophlebiidae: Ephemeroptera). Florida Entomol. 52: 253-258.

PETERS, W.L. 1971. A revision of the Leptophlebiidae of the West Indies (Ephemeroptera). Smith. Contr. Zool. 62: $1-48$.

PETERS, W.L. 1981. Coryphorus aquilus, a new genus and species of Tricorythidae from the Amazon Basin (Ephemeroptera). Aquatic Insects. 3: 209-217.

PETERS, W.L. \& EDMUNDS JR, G.F. 1972. A revision of the generic classification of certain Leptophlebiidae from southern South America (Ephemeroptera). Ann. Entomol. Soc. Amer. 65: 1398-1414.

PICTET, F.J. (1843-1845). Histoire naturelle générale et particulière des insectes névroptères. Famille des éphémérines. Geneva.

PUTHZ, V. 1975. Eine neue Caenidengattung aus dem Amazonasgebiet (Insecta: Ephemeroptera: Caenidae). Amazoniana. 5: 411-415. 
SALLES, F.F. \& BATISTA, J.D. 2004. The presence of Varipes Lugo-Ortiz \& McCafferty (Ephemeroptera: Baetidae) in Brazil, with the description of a new species. Zootaxa 456: 1-6.

SALLES, F.F., BATISTA, J.D. \& CABETTE, H.R.S. 2004 b. Baetidae (Insecta: Ephemeroptera) de Nova Xavantina, Mato Grosso, Brasil: novos registros e descrição de uma nova espécie de Cloeodes Traver. Biota Neotrop. 4(2): 1-8.

SALLES, F.F., DA-SILVA, E.R. \& LUGO-ORTIZ, C.R. 2003a. Descrição da ninfa e redescrição dos adultos de Callibaetis radiatus Navás (Insecta: Ephemeroptera: Baetidae). Lundiana 4(1): 13-18.

SALLES, F.F., DA-SILVA, E.R., SERRÃO, J.E. \& FRANCISCHETTI, C.N. aceito. Baetidae (Ephemeroptera) na Região Sudeste do Brasil: novos registros e chave para os gêneros no estágio ninfal. Neotrop. Entomol.

SALLES, F.F. \& DIAS, L.G. aceito. Descrição dos adultos de Camelobaetidius billi (Ephemeroptera, Baetidae). Iheringia S. Zool.

SALLES, F.F. \& FRANCISCHETTI, C.N. 2004. Cryptonympha dasilvai sp. nov. (Ephemeroptera: Baetidae) do Brasil. Neotrop. Entomol. 33(2): 213-216.

SALLES, F.F., FRANCISCHETTI, C.N., ROQUE, F.O., PEPINELLI, M. \& STRIXINO, S.T. 2003b. Levantamento preliminar dos gêneros e espécies de Baetidae (Insecta: Ephemeroptera) do Estado de São Paulo, com ênfase em coletas realizadas em córregos florestados de baixa ordem. Biota Neotrop. 3(2): 1-7.

SALLES, F.F. \& LUGO-ORTIZ, C.R. 2002a. A distinctive new species of Apobaetis (Ephemeroptera: Baetidae) from Mato Grosso and Minas Gerais, Brazil. Zootaxa 35: 1-6.

SALLES, F.F. \& LUGO-ORTIZ, C.R. 2002b. Primeiro registro do gênero Harpagobaetis Mol (Ephemeroptera: Baetidae) para o Brasil. Lundiana 3: 155.

SALLES, F.F. \& LUGO-ORTIZ, C.R. 2003a. Nova espécie de Cloeodes Traver (Ephemeorptera: Baetidae) do Estado do Rio de Janeiro. Neotrop. Entomol. 32(3): 449-452.

SALLES, F.F. \& LUGO-ORTIZ, C.R. 2003b. Um novo gênero e espécie de Baetidae (Ephemeroptera) do Estado de Minas Gerais, sudeste do Brasil. Iheringia S. Zool. 93(2): 201-206.

SALLES, F.F., LUGO-ORTIZ, C.R. \& DA-SILVA, E.R. 2004a. Descrição da fêmea adulta de Americabaetis titthion (Ephemeroptera: Baetidae). Acta Zool. Mex. 20(1): 23-26.

SALLES, F.F., LUGO-ORTIZ, C.R., DA-SILVA, E.R. \& FRANCISCHETTI, C.N. 2003c. Novo gênero e espécie de Baetidae (Insecta, Ephemeroptera) do Brasil. Arq. Mus. Nac. 61(1): 23-30.
SAVAGE, H.M. 1982. A curious new genus and species of Atalophlebiinae (Ephemeroptera: Leptophlebiidae) from the southern coastal mountains of Brazil. Stud. Neotrop. Fauna Environ. 17: 209-217.

SAVAGE, H.M. 1983. Perissophlebiodes, a Replacement Name for Perissophlebia Savage Nec Tillyard (Ephemeroptera, Leptophlebiidae). Entomol. News. 94(5): 204-204.

SAVAGE, H.M. 1986. Systematics of the Terpides lineage from the Neotropics: Definition of the Terpides lineage, methods, and revision of Fittkaulus Savage \& Peters. Spixiana 9: 255-270.

SAVAGE, H.M. \& DOMÍNGUEZ, E. 1992. A new genus of Atalophlebiinae (Ephemeroptera, Leptophlebiidae) from northern South America. Aquatic Insects. 14: 243-248.

SAVAGE, H.M. \& PETERS, W.L. 1978. Fittkaulus maculatus, a new genus and species from northern Brazil (Leptophlebiidae: Ephemeroptera). Acta Amazonica. 8: 293-298.

SAVAGE, H.M. \& PETERS, W.L. 1983. Systematics of Miroculis and related genera from northern South America (Ephemeroptera: Leptophlebiidae). Trans. Amer. Entomol. Soc. 108: 491-600.

SOLDÁN, T. 1986. A revision of the Caenidae with ocellar tubercles in the nymphal stage (Ephemeroptera). Acta Univers. Carol. Biol. 1982-1984: 289-362.

SPIETH, H.T. 1943. Taxonomic studies on the Ephemeroptera. III. Some interesting Ephemerids from Surinam and other Neotropical localities. Amer. Mus. Novit. 1244: 1-13.

STEPHENS, J.F. 1835. Ilustration of British Entomology, Mandibulata. 6: 54-70.

THEW, T.B. 1960. Taxonomic studies on some Neotropical Leptophlebiid mayflies (Ephemeroptera: Leptophlebiidae). Pan-Pac. Entomol. 36: 119-132.

TRAVER, J.R. 1938. Mayflies of Puerto Rico. J. Agric. Univ. Puerto Rico 22: 5-42.

TRAVER, J.R. 1944. Notes on Brazilian mayflies. Bol. Mus. Nac., N.S. Zoo. 22: 2-53.

TRAVER, J.R. 1946. Notes on Neotropical mayflies. Part I. Family Baetidae, subfamily Leptophlebiinae. Rev. Entomol. 17: 418-436.

TRAVER, J.R. 1950. Notes on Neotropical mayflies. Part. IV. Family Ephemeridae (continued). Rev. Entomol. 21: 593614.

TRAVER, J.R. 1956. A new genus of Neotropical mayflies (Ephemeroptera, Leptophlebiidae). Proc. Entomol. Soc. Washington. 58: 1-13.

TRAVER, J.R. 1958. The subfamily Leptohyphinae (Ephemeroptera: Tricorythidae). Part I. Ann. Entomol. Soc. Amer. 51: 491-503. 
TRAVER, J.R. 1959. Uruguayan mayflies. Family Leptophlebiidae: Part I. Rev. Soc. Urug. Entomol. 3: 1-13.

TRAVER, J.R. 1960. Uruguayan mayflies. Family Leptophlebiidae: Part III. Rev. Soc. Urug. Entomol. 4:7385.

TRAVER, J.R. \& EDMUNDS JR, G.F. 1967. A revision of the genus Thraulodes (Ephemeroptera: Leptophlebiidae). Misc. Pub. Entomol. Soc. Am. 85(11): 1-80.

TRAVER, J.R. \& EDMUNDS JR, G.F. 1968. A revision of the Baetidae with spatulate-clawed nymphs (Ephemeroptera). Pac. Insects 10: 629-677.

ULMER, G. 1920a. Neue Ephemeropteren. Arch. Naturgesch. 85(11): 1-80.

ULMER, G. 1921. Über einige Ephemeropteren-Typen älterer Autoren. Arch. Naturgesch. 87: 229-237.

ULMER, G. 1942. Alte und neue Eintagsfliegen (Ephemeropteren) aus Süd- und Mittelamerika. Stett. Entomol. Z. 103: 98-128.

ULMER, G. 1943. Alte und neue Eintagsfliegen (Ephemeropteren) aus Süd- und Mittelamerika. Stett. Entomol. Z. 104: 14-46.

WALKER, F. 1853. Ephemerinae. List of the specimens of neuropterous insects in the collection of the British Museum, Part III (Termitidae- Ephemeridae). 533-585.

WALKER, F. 1860. Characters of undescribed Neuroptera in the Collection of W.W. Saunders, Esq. F.R.S. Trans. Entomol. Soc. London. 5: 176-199.

WALSH, B.D. 1863. Observations on certain N.A. Neuroptera, by H. Hagen, M.D., of Koeningsberg, Prussia; translated from the original French MS, and published by permission of the author, with notes and descriptions of about twenty new N.A. species of Pseudoneuroptera. Proc. Entomol. Soc. Philadelphia. 2: 167-272.

WALTZ, R.D. \& McCAFFERTY, W.P. 1985. Moribaetis: A new genus of Neotropical Baetidae (Ephemeroptera). Proc. Entomol. Soc. Washington. 87: 239-251.

WEBER, F. 1801. Observationes Entomologicae. 99-100.

WIERSEMA, N.A. \& McCAFFERTY, W.P. 2000. Generic revision of the North and Central American Leptohyphidae (Ephemeroptera: Pannota).Trans. Am. Entomol. Soc. 126: 337-371.

WILLIAMSON, H. 1802. On the Ephoron leukon, usually called the white fly of the Passaick River. Trans. Am. Phil. Soc. 5: 71-73.
Title: As espécies de Ephemeroptera (Insecta) registradas para o Brasil

Authors: Frederico Falcão Salles; Elidiomar Ribeiro DaSilva; Michael D. Hubbard; José Eduardo Serrão

Biota Neotropica, Vol. 4 ( number 2): 2004

http://www.biotaneotropica.org.br/v4n2/pt/ abstract?inventory+BN04004022004

Date Received 07/11/2004

Accepted 10/27/2004

ISSN 1676-0603 
Tabela 1. Lista das espécies de Ephemeroptera registradas para o Brasil, acompanhada da distribuição por estado e bibliografia referente aos registros.

\begin{tabular}{|c|c|c|}
\hline \multicolumn{3}{|c|}{ FAMÍLIA BAETIDAE (20 gêneros, 46 espécies) } \\
\hline \multicolumn{3}{|l|}{ Adebrotus Lugo-Ortiz \& McCafferty, 1995} \\
\hline $\begin{array}{l}\text { Adebrotus amazonicus Lugo-Ortiz \& } \\
\text { McCafferty, } 1995\end{array}$ & [MT; AM] & $\begin{array}{l}\text { (Lugo-Ortiz \& McCafferty 1995, Salles et } \\
\text { al. 2004b) }\end{array}$ \\
\hline \multicolumn{3}{|l|}{ Americabaetis Kluge, 1992} \\
\hline $\begin{array}{l}\text { Americabaetis alphus Lugo-Ortiz \& } \\
\text { McCafferty, } 1996\end{array}$ & $\begin{array}{c}\text { [PR, SC, RS; MG, } \\
\text { RJ, SP; MT] }\end{array}$ & $\begin{array}{l}\text { (Lugo-Ortiz \& McCafferty 1996c, } \\
\text { Francischetti et al. 2003, Salles et al. } \\
\text { 2003c, aceito a, b) }\end{array}$ \\
\hline $\begin{array}{l}\text { Americabaetis labiosus Lugo-Ortiz \& } \\
\text { McCafferty, } 1996\end{array}$ & [PR, SC, RS; RJ] & $\begin{array}{l}\text { (Lugo-Ortiz \& McCafferty 1996c, } \\
\text { Francischetti et al. 2003, Salles et al. aceito } \\
\text { a) }\end{array}$ \\
\hline $\begin{array}{l}\text { Americabaetis longetron Lugo-Ortiz \& } \\
\text { McCafferty, } 1996\end{array}$ & $\begin{array}{c}\text { [PR, SC; ES, RJ, } \\
\text { MG] }\end{array}$ & $\begin{array}{l}\text { (Lugo-Ortiz \& McCafferty 1996c, } \\
\text { Francischetti et al. 2003, Salles et al. aceito } \\
\text { a) }\end{array}$ \\
\hline $\begin{array}{l}\text { Americabaetis titthion Lugo-Ortiz \& } \\
\text { McCafferty, } 1996\end{array}$ & [PR, SC, RS; RJ] & $\begin{array}{l}\text { (Lugo-Ortiz \& McCafferty 1996c, } \\
\text { Francischetti et al. 2003, Salles et al. 2004, } \\
\text { aceito a) }\end{array}$ \\
\hline \multicolumn{3}{|l|}{ Apobaetis Day, 1955} \\
\hline Apobaetis fiuzai Salles \& Lugo-Ortiz, 2002a & $\begin{array}{c}\text { [MG, RJ, SP; } \\
\text { MT] }\end{array}$ & $\begin{array}{l}\text { (Salles \& Lugo-Ortiz 2002a, Salles et al. } \\
\text { aceito a) }\end{array}$ \\
\hline $\begin{array}{l}\text { Apobaetis signifer Lugo-Ortiz \& } \\
\text { McCafferty, } 1997\end{array}$ & {$[\mathrm{PA}]$} & (Lugo-Ortiz \& McCafferty 1997) \\
\hline \multicolumn{3}{|l|}{ Aturbina Lugo-Ortiz \& McCafferty, 1996} \\
\hline Aturbina georgei Lugo-Ortiz \& McCafferty, & [SP, MG, RJ; & (Lugo-Ortiz \& McCafferty 1996a, Salles et \\
\hline
\end{tabular}




\begin{tabular}{|c|c|c|}
\hline 1996 & MT; AM, PA] & al. 2003c, aceito a, b, Nolte et al. 1997) \\
\hline \multicolumn{3}{|l|}{ Baetodes Needham \& Murphy, 1924} \\
\hline Baetodes itatiayanus Demoulin, 1955 & {$[\mathrm{RJ}]$} & (Demoulin 1955) \\
\hline Baetodes sancticatarinae Mayo, 1968 & [SC] & (Mayo 1968) \\
\hline $\begin{array}{l}\text { Baetodes serratus Needham \& Murphy, } \\
1924\end{array}$ & {$[\mathrm{MG}, \mathrm{RJ}]$} & (Needham \& Murphy 1924, Traver 1944) \\
\hline \multicolumn{3}{|l|}{ Callibaetis Eaton, 1881} \\
\hline $\begin{array}{l}\text { Callibaetis fasciatus (Pictet), } 1843 \\
=\text { Cloe fasciata Pictet, } 1843 \\
=\text { Cloeon fasciata (Pictet, 1843) } \\
=\text { Baetis fasciata (Pictet, 1843) } \\
=\text { Callibaetis trifasciatus Esben-Petersen, } \\
1912 \\
=\text { Baetis gloriosus Navás, 1923 } \\
=\text { Callibaetis gloriosus (Navás, 1923) }\end{array}$ & [??] & (Pictet 1843) \\
\hline Callibaetis gregarius Navás, 1930b & [SP] & (Navás 1930b) \\
\hline $\begin{array}{l}\text { Callibaetis guttatus Navás, 1915a } \\
=\text { Callibaetis apicatus Navás, } 1917 \\
=\text { Callibaetis bruchius Navás, 1920b } \\
=\text { Callibaetis zonatus Navás, } 1929\end{array}$ & {$[\mathrm{RJ}]$} & (Da-Silva 1991) \\
\hline
\end{tabular}




\begin{tabular}{|c|c|c|}
\hline $\begin{array}{l}\text { Callibaetis jocosus Navás, } 1912 \\
=\text { Callibaetis strictogaster Navás, 1915a } \\
=\text { Callibaetis jaffueli Navás, } 1918 \\
=\text { Callibaetis spegazzinus Navás, 1920b } \\
=\text { Callibaetis rimatus Navás, } 1932\end{array}$ & [SP] & (Navás 1912) \\
\hline $\begin{array}{l}\text { Callibaetis pollens Neddham \& Murphy, } \\
1924\end{array}$ & [MS] & (Needham \& Murphy 1924) \\
\hline $\begin{array}{l}\text { Callibaetis radiatus Navás, 1920a } \\
\text { =Callibaetis venulosus Navás, } 1932\end{array}$ & [MG] & (Salles et al. 2003b) \\
\hline $\begin{array}{l}\text { Callibaetis viviparus Needham \& Murphy, } \\
1924\end{array}$ & [MS] & (Needham \& Murphy 1924) \\
\hline $\begin{array}{l}\text { Callibaetis willineri Navás, } 1932 \\
=\text { Callibaetis alegre } \text { Traver, } 1944\end{array}$ & [PR, RS] & (Traver 1944, Melo et al. 2002) \\
\hline $\begin{array}{l}\text { Callibaetis zonalis Navás, 1915b } \\
=\text { Callibaetis vitreus Navás, 1915a } \\
=\text { Baetis opacus Navás, 1915b } \\
=\text { Callibaetis sobrius Navás, } 1916 \\
=\text { Baetis virellus Navás, 1915a } \\
=\text { Callibaetis apertus Navás, } 1917 \\
=\text { Callibaetis vitreus Navás, } 1919 \\
=\text { Callibaetis depressus Navás, } 1922 \\
=\text { Callibaetis amoenus Navás, 1930a }\end{array}$ & {$[\mathrm{SP}]$} & (Navás 1916) \\
\hline
\end{tabular}




\begin{tabular}{|c|c|c|}
\hline $\begin{array}{l}\text { Camelobaetidius anubis (Traver \& } \\
\text { Edmunds, 1968) } \\
\text { =Dactylobaetis anubis Traver \& Edmunds, } \\
1968\end{array}$ & $\begin{array}{c}\text { [PR, SC; SP, MG, } \\
\text { RJ] }\end{array}$ & $\begin{array}{l}\text { (Traver \& Edmunds 1968, Salles et al. } \\
\text { 2003c, aceito a) }\end{array}$ \\
\hline $\begin{array}{l}\text { Camelobaetidius billi Thomas \& } \\
\text { Dominique, } 2000\end{array}$ & [AM] & (Salles \& Dias, aceito) \\
\hline $\begin{array}{l}\text { Camelobaetidius janae Dominique \& } \\
\text { Thomas, } 2000\end{array}$ & [MT] & (Salles et al. 2004b) \\
\hline $\begin{array}{l}\text { Camelobaetidius mantis Traver \& } \\
\text { Edmunds, } 1968\end{array}$ & {$[\mathrm{AM}]$} & (Traver \& Edmunds 1968) \\
\hline $\begin{array}{l}\text { Camelobaetidius phaedrus (Traver \& } \\
\text { Edmunds, 1968) } \\
\text { =Dactylobaetis phaedrus Traver \& } \\
\text { Edmunds, } 1968\end{array}$ & {$[\mathrm{RS}, \mathrm{SC}]$} & (Traver \& Edmunds 1968) \\
\hline $\begin{array}{l}\text { Camelobaetidius serapis (Traver \& } \\
\text { Edmunds, 1968) } \\
\text { =Dactylobaetis serapis Traver \& Edmunds, } \\
1968\end{array}$ & [SC] & (Traver \& Edmunds 1968) \\
\hline Cloeodes Traver, 1938 & & \\
\hline $\begin{array}{l}\text { Cloeodes hydation McCafferty \& Lugo- } \\
\text { Ortiz, } 1995\end{array}$ & [MG; MT] & $\begin{array}{l}\text { (McCafferty \& Lugo-Ortiz 1995, Salles et } \\
\text { al. aceito a) }\end{array}$ \\
\hline Cloeodes irvingi Waltz \& McCafferty, 1987 & [ES, MG, RJ, SP] & $\begin{array}{l}\text { (Lugo-Ortiz et al. 2002, Salles et al. 2003c, } \\
\text { aceito a) }\end{array}$ \\
\hline $\begin{array}{l}\text { Cloeodes jaragua Salles \& Lugo-Ortiz, } \\
\text { 2003a }\end{array}$ & [RJ] & (Salles \& Lugo-Ortiz 2003b) \\
\hline Cloeodes auwe Salles \& Batista, 2004 & [MT] & (Salles et al. 2004b) \\
\hline \multicolumn{3}{|l|}{ Cryptonympha Lugo-Ortiz \& McCafferty, 1998} \\
\hline Cryptonympha copiosa Lugo-Ortiz \& & [RS, SC; AC, & (Lugo-Ortiz \& McCafferty 1998) \\
\hline
\end{tabular}




\begin{tabular}{|c|c|c|}
\hline McCafferty, 1998 & AM, PA] & \\
\hline $\begin{array}{l}\text { Cryptonympha dasilvai Salles \& } \\
\text { Francischetti, } 2004\end{array}$ & {$[\mathrm{RJ}, \mathrm{SP}]$} & (Salles \& Francischetti 2004) \\
\hline \multicolumn{3}{|l|}{ Harpagobaetis Mol, 1986} \\
\hline Harpagobaetis gulosus Mol, 1986 & {$[\mathrm{GO}, \mathrm{MT}]$} & $\begin{array}{l}\text { (Salles \& Lugo-Ortiz 2002b, Salles et al. } \\
\text { 2004b) }\end{array}$ \\
\hline \multicolumn{3}{|l|}{ Iguaira Salles \& Lugo-Ortiz, 2003b } \\
\hline $\begin{array}{l}\text { Iguaira poranga Salles \& Lugo-Ortiz, } \\
\text { 2003b }\end{array}$ & {$[\mathrm{MG}]$} & (Salles \& Lugo-Ortiz 2003a) \\
\hline \multicolumn{3}{|l|}{ Moribaetis Waltz \& McCafferty, 1985} \\
\hline $\begin{array}{l}\text { Moribaetis comes (Navás, 1912) } \\
=\text { Baetis comes Navás, } 1912\end{array}$ & [SP] & (Navás 1912a) \\
\hline \multicolumn{3}{|l|}{ Paracloeodes Day, 1955} \\
\hline $\begin{array}{l}\text { Paracloeodes binodulus Lugo-Ortiz \& } \\
\text { McCafferty, } 1996\end{array}$ & [MT; PA] & $\begin{array}{l}\text { (Lugo-Ortiz \& McCafferty 1996b, Salles et } \\
\text { al. 2004b) }\end{array}$ \\
\hline $\begin{array}{l}\text { Paracloeodes eurybranchus Lugo-Ortiz \& } \\
\text { McCafferty, } 1996\end{array}$ & $\begin{array}{c}\text { [RS; ES, RJ, MG, } \\
\text { SP] }\end{array}$ & $\begin{array}{l}\text { (Lugo-Ortiz \& McCafferty 1996b, Lugo- } \\
\text { Ortiz et al. 2002, Salles et al. 2003c, aceito } \\
\text { a) }\end{array}$ \\
\hline $\begin{array}{l}\text { Paracloeodes leptobranchus Lugo-Ortiz \& } \\
\text { McCafferty, } 1996\end{array}$ & [PR, SC, RS] & (Lugo-Ortiz \& McCafferty 1996b) \\
\hline \multicolumn{3}{|l|}{ Rivudiva Lugo-Ortiz \& McCafferty, 1998} \\
\hline $\begin{array}{l}\text { Rivudiva minantenna Lugo-Ortiz \& } \\
\text { McCafferty, } 1998\end{array}$ & {$[\mathrm{RS}, \mathrm{SC} ; \mathrm{RJ}]$} & $\begin{array}{l}\text { (Lugo-Ortiz \& McCafferty 1998, Salles et } \\
\text { al. aceito a) }\end{array}$ \\
\hline Rivudiva trichobasis Lugo-Ortiz \& & [RS] & (Lugo-Ortiz \& McCafferty 1998) \\
\hline
\end{tabular}




\begin{tabular}{|c|c|c|}
\hline McCafferty, 1998 & & \\
\hline Spiritiops Lugo-Ortiz \& McCafferty, 1998 & & \\
\hline $\begin{array}{l}\text { Spiritiops silvudus Lugo-Ortiz \& } \\
\text { McCafferty, } 1998\end{array}$ & [MT; AM, PA] & $\begin{array}{l}\text { (Lugo-Ortiz \& McCafferty 1998, Salles et } \\
\text { al. 2004b) }\end{array}$ \\
\hline Tomedontus Lugo-Ortiz \& McCafferty, 1995 & & \\
\hline $\begin{array}{l}\text { Tomedontus primus Lugo-Ortiz \& } \\
\text { McCafferty, } 1995\end{array}$ & {$[\mathrm{AM}]$} & (Lugo-Ortiz \& McCafferty 1995) \\
\hline Tupiara Salles, Lugo-Ortiz, Da-Silva \& Fran & ischetti, 2003 & \\
\hline $\begin{array}{l}\text { Tupiara ibirapitanga Salles, Lugo-Ortiz, } \\
\text { Da-Silva \& Francischetti, } 2003\end{array}$ & [AM; MG, RJ] & (Salles et al. 2003a) \\
\hline Waltzoyphius McCafferty \& Lugo-Ortiz, 199 & & \\
\hline $\begin{array}{l}\text { Waltzoyphius fasciatus McCafferty \& Lugo- } \\
\text { Ortiz, } 1995\end{array}$ & $\begin{array}{c}\text { [ES, MG, RJ, SP; } \\
\text { MT; AM, PA] }\end{array}$ & $\begin{array}{l}\text { (Lugo-Ortiz \& McCafferty 1995, Lugo- } \\
\text { Ortiz et al. 2002, Salles et al. 2003c, aceito } \\
\text { a, b) }\end{array}$ \\
\hline Varipes Lugo-Ortiz \& McCafferty, 1998 & & \\
\hline Varipes helenae Salles \& Batista, 2004 & {$[\mathrm{MT}]$} & (Salles \& Batista 2004) \\
\hline Zelusia Lugo-Ortiz \& McCafferty, 1998 & & \\
\hline $\begin{array}{l}\text { Zelusia principalis Lugo-Ortiz \& } \\
\text { McCafferty, } 1998\end{array}$ & $\begin{array}{c}\text { [ES, MG, RJ, SP; } \\
\text { MT; AM, PA] }\end{array}$ & $\begin{array}{l}\text { (Lugo-Ortiz \& McCafferty 1998, Lugo- } \\
\text { Ortiz et al. 2002, Salles et al. 2003c, aceito } \\
\text { a, b) }\end{array}$ \\
\hline FAMÍLIA CA & ENIDAE (3 gênero & s, 15 espécies) \\
\hline
\end{tabular}




\begin{tabular}{|c|c|c|}
\hline \multicolumn{3}{|l|}{ Brasilocaenis Puthz, 1975} \\
\hline $\begin{array}{l}\text { Brasilocaenis intermedia } \\
\text { Malzacher, } 1986\end{array}$ & [REGIÃO NORTE] & (Malzacher 1986) \\
\hline Brasilocaenis irmleri Puthz, 1975 & [MT; AM] & (Puthz 1975, Nolte et al. 1997) \\
\hline $\begin{array}{l}\text { Brasilocaenis mendesi Malzacher, } \\
1998\end{array}$ & [REGIÃO CENTRO-OESTE] & (Malzacher 1998) \\
\hline $\begin{array}{l}\text { Brasilocaenis puthzi Malzacher, } \\
1986\end{array}$ & [MT; AM] & $\begin{array}{l}\text { (Malzacher 1986, Nolte et al. } \\
\text { 1997) }\end{array}$ \\
\hline $\begin{array}{l}\text { Brasilocaenis renata Malzacher, } \\
1986\end{array}$ & {$[\mathrm{AM}]$} & (Malzacher 1986) \\
\hline $\begin{array}{l}\text { Brasilocaenis septentrionalis } \\
\text { Malzacher, } 1990\end{array}$ & {$[\mathrm{PA}]$} & (Malzacher 1990) \\
\hline \multicolumn{3}{|l|}{ Caenis Stephens, 1835} \\
\hline Caenis candelata Malzacher, 1986 & {$[\mathrm{AM}]$} & (Malzacher 1986) \\
\hline $\begin{array}{l}\text { Caenis cigana Pereira \& Da-Silva, } \\
\text { 1990a }\end{array}$ & {$[\mathrm{RJ}]$} & (Pereira \& Da-Silva 1990a) \\
\hline Caenis cuniana Froehlich, 1969 & {$[\mathrm{RJ}, \mathrm{SP}]$} & (Froehlich 1969, Da-Silva 1993a) \\
\hline \begin{tabular}{|l|} 
Caenis fittkaui Malzacher, 1986 \\
\end{tabular} & {$[\mathrm{PA}]$} & (Malzacher 1986) \\
\hline $\begin{array}{l}\text { Caenis pflugfelderi Malzacher, } \\
1990\end{array}$ & {$[\mathrm{AM}]$} & (Malzacher 1990) \\
\hline $\begin{array}{l}\text { Caenis quatipuruica Malzacher, } \\
1986\end{array}$ & {$[\mathrm{PA}]$} & (Malzacher 1986) \\
\hline Caenis reissi Malzacher, 1986 & {$[\mathrm{PA}]$} & (Malzacher 1986) \\
\hline \begin{tabular}{|l|} 
Caenis sigillata Malzacher, 1986 \\
\end{tabular} & [REGIÃO NORTE] & (Malzacher 1986) \\
\hline \multicolumn{3}{|l|}{ Cercobrachys Soldán, 1986} \\
\hline $\begin{array}{l}\text { Cercobrachys columbianus Soldán, } \\
1986\end{array}$ & [AM] & (Malzacher 1986) \\
\hline
\end{tabular}




\begin{tabular}{|c|c|c|}
\hline \multicolumn{3}{|c|}{ FAMÍLIA CORYPHORIDAE (1 gênero, 1 espécie) } \\
\hline \multicolumn{3}{|l|}{ Coryphorus Peters, 1981} \\
\hline Coryphorus aquilus Peters, 1981 & {$[\mathrm{AM}, \mathrm{PA}]$} & (Peters 1981) \\
\hline \multicolumn{3}{|c|}{ FAMÍLIA EPHEMERIDAE (1 gênero, 1 espécie) } \\
\hline \multicolumn{3}{|l|}{ Hexagenia Walsh, 1863} \\
\hline $\begin{array}{l}\text { Hexagenia (Pseudeatonica) } \\
\text { albivitta (Walker, 1853) } \\
=\text { Baetis albivitta Walker, } 1853 \\
=\text { Eatonica (Pseudeatonica) } \\
\text { albivitta (Walker, 1853) } \\
=\text { Palingenia continua Walker, } \\
1860 \\
=\text { Palingenia dorsigera Hagen, } \\
1861 \text { (nomem nudum) } \\
=\text { Hexagenia benedicta Navás, } \\
1922 \\
=\text { Hexagenia dominans Navás, } \\
1936\end{array}$ & [PR; SP; PA] & $\begin{array}{l}\text { (Walker 1853, Navás 1922a, } \\
\text { 1936b, McCafferty 1970) }\end{array}$ \\
\hline \multicolumn{3}{|c|}{ FAMÍLIA EUTHYPLOCIIDAE (2 gêneros, 4 espécies) } \\
\hline Campylocia Needham \& Murphy, & & \\
\hline
\end{tabular}




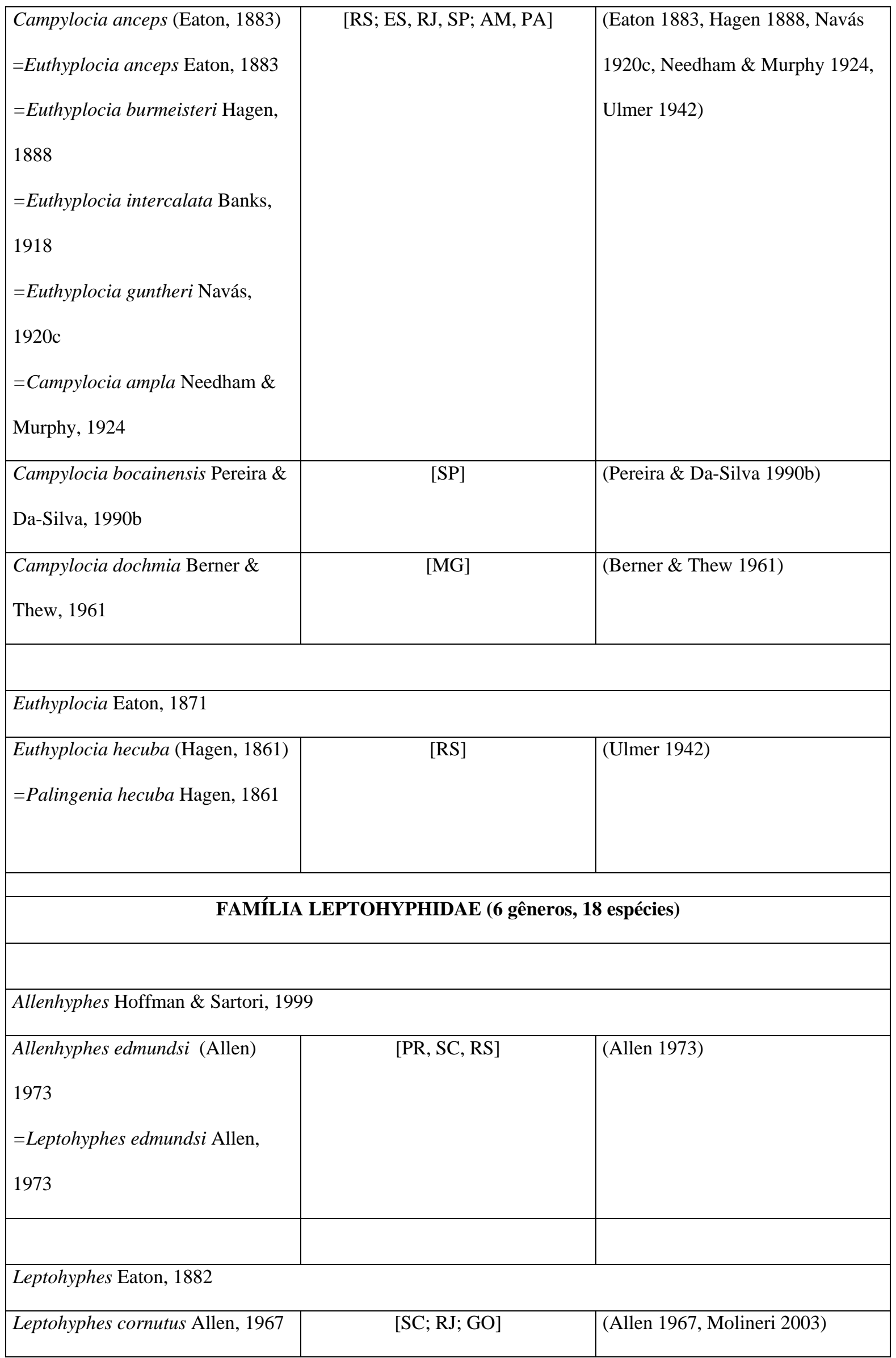




\begin{tabular}{|c|c|c|}
\hline $\begin{array}{l}\text { Leptohyphes mollipes Needham \& } \\
\text { Murphy, } 1924\end{array}$ & [??] & (Needham \& Murphy 1924) \\
\hline $\begin{array}{l}\text { Leptohyphes peterseni Ulmer, } \\
1920\end{array}$ & {$[\mathrm{SC}]$} & (Ulmer 1920) \\
\hline $\begin{array}{l}\text { Leptohyphes plaumanni Allen, } \\
1967 \\
=\text { Leptohyphes pereirae Da-Silva, } \\
\text { 1993b }\end{array}$ & [SC; RJ] & (Allen 1967, Da-Silva 1993b) \\
\hline Leptohyphes populus Allen, 1973 & {$[\mathrm{AM}]$} & (Allen 1973) \\
\hline \multicolumn{3}{|l|}{ Leptohyphodes Ulmer, 1920} \\
\hline $\begin{array}{l}\text { Leptohyphodes inanis (Pictet, } \\
\text { 1843) } \\
\text { =Potamanthus? inanis Pictet, } 1843\end{array}$ & [??] & (Pictet 1843) \\
\hline \multicolumn{3}{|l|}{ Traverhyphes Molineri, 2001} \\
\hline Traverhyphes pirai Molineri, 2001 & {$[\mathrm{RJ}]$} & (Molineri 2001a) \\
\hline \multicolumn{3}{|l|}{ Tricorythodes Ulmer, 1920} \\
\hline $\begin{array}{l}\text { Tricorythodes arequita Molineri, } \\
2002\end{array}$ & [RS] & (Molineri 2002) \\
\hline $\begin{array}{l}\text { Tricorythodes australis (Banks, } \\
\text { 1913) } \\
=\text { Tricorythus australis Banks, } \\
1913 \\
=\text { Leptohyphodes australis (Banks, } \\
\text { 1913) }\end{array}$ & [PR; MT; PA] & (Molineri 2002) \\
\hline
\end{tabular}




\begin{tabular}{|c|c|c|}
\hline $\begin{array}{l}\text { Tricorythodes barbus Allen } 1967 \\
=\text { Tricorythodes (Tricorythodes) } \\
\text { barbus; Allen \& Murvosh } 1987 \\
=\text { Tricorhyphes barbus; Wiersema } \\
\text { \& McCafferty } 2000\end{array}$ & [SC] & (Allen 1967) \\
\hline $\begin{array}{l}\text { Tricorythodes bullus Allen, } 1967 \\
=\text { Tricorythodes (Tricorythodes) } \\
\text { bullus; Allen \& Murvosh } 1987 \\
=\text { Epiphrades bullus; Wiersema \& } \\
\text { McCafferty } 2000\end{array}$ & {$[\mathrm{SC}]$} & (Allen 1967) \\
\hline $\begin{array}{l}\text { Tricorythodes cristatus Allen, } \\
1967 \\
=\text { Tricorythodes (Tricorythodes) } \\
\text { cristatus; Allen \& Murvosh } 1987 \\
=\text { Epiphrades cristatus; Wiersema } \\
\text { \& McCafferty } 2000\end{array}$ & [REGIÃO SUDESTE] & (Allen 1967) \\
\hline \multicolumn{3}{|l|}{ Tricorythopsis Traver, 1958} \\
\hline $\begin{array}{l}\text { Tricorythopsis artigas Traver, } \\
1958 \\
=\text { Leptohyphes tinctus Allen, } 1973 \\
=\text { Tricorythopsis fictilis Molineri, } \\
1999 \\
=\text { Allenhyphes tinctus; Wiersema \& } \\
\text { McCafferty } 2000\end{array}$ & [RS] & (Allen 1973) \\
\hline $\begin{array}{l}\text { Tricorythopsis gibbus (Allen, } \\
\text { 1967) } \\
\text { =Leptohyphes gibbus Allen, } 1967\end{array}$ & [SC] & (Allen 1967) \\
\hline
\end{tabular}




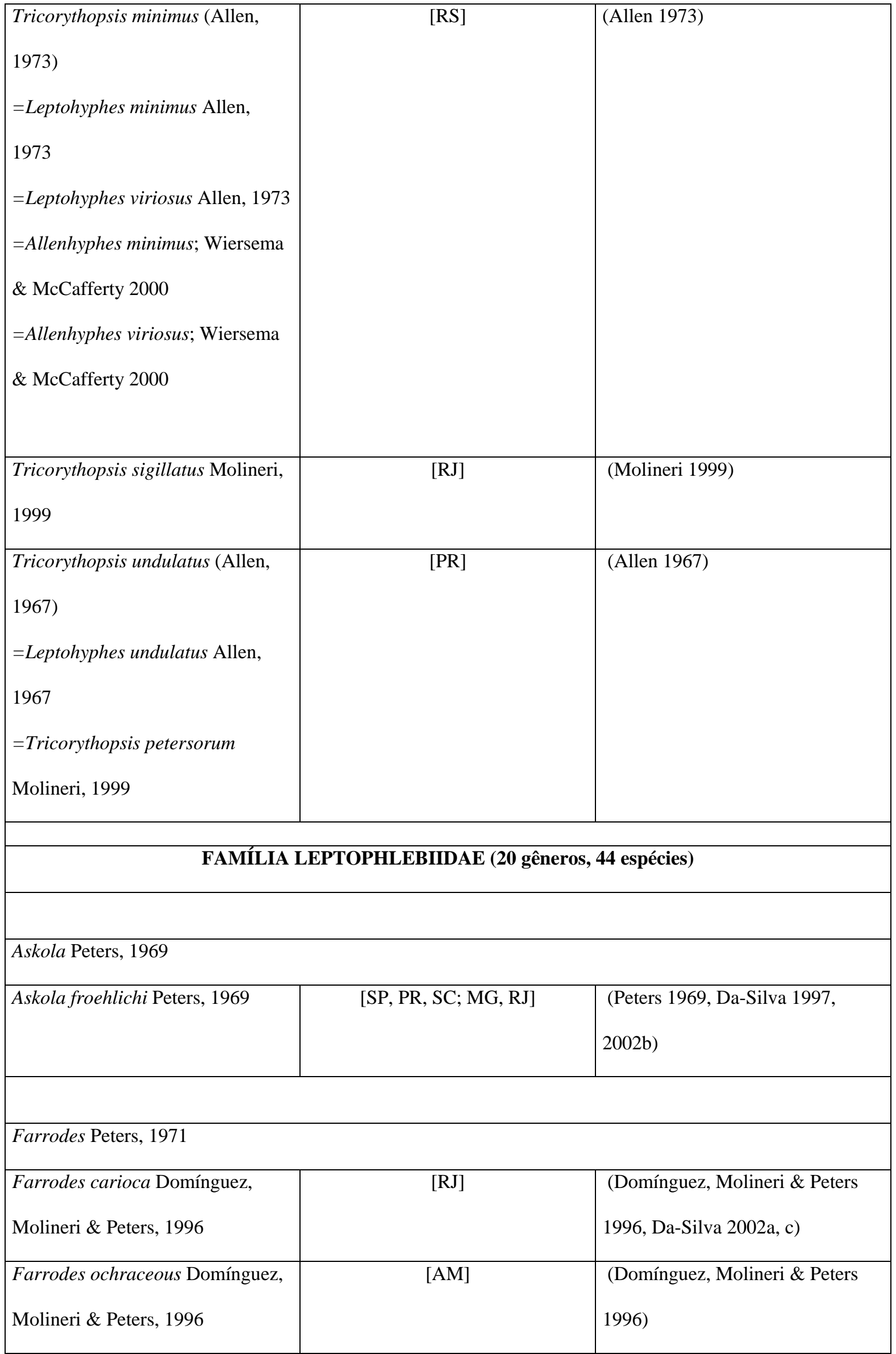




\begin{tabular}{|c|c|c|}
\hline $\begin{array}{l}\text { Farrodes xingu Domínguez, } \\
\text { Molineri \& Peters, } 1996\end{array}$ & {$[\mathrm{PA}]$} & $\begin{array}{l}\text { (Domínguez, Molineri \& Peters } \\
1996)\end{array}$ \\
\hline \multicolumn{3}{|l|}{ Fittkaulus Savage \& Peters, 1978} \\
\hline Fittkaulus cuiabae Savage, 1986 & {$[\mathrm{MT}]$} & (Savage 1986) \\
\hline $\begin{array}{l}\text { Fittkaulus cururuensis Savage, } \\
1986\end{array}$ & {$[\mathrm{PA}]$} & (Savage 1986) \\
\hline $\begin{array}{l}\text { Fittkaulus maculatus Savage \& } \\
\text { Peters, } 1978\end{array}$ & [BA; PA] & $\begin{array}{l}\text { (Savage \& Peters 1978, Da-Silva } \\
\text { 1992) }\end{array}$ \\
\hline \multicolumn{3}{|l|}{ Hagenulopsis Ulmer, 1920} \\
\hline Hagenulopsis diptera Ulmer, 1920 & {$[\mathrm{SC}]$} & (Ulmer 1920) \\
\hline \multicolumn{3}{|c|}{ Hermanella Needham \& Murphy, 1924} \\
\hline $\begin{array}{l}\text { Hermanella (Guayakia) froehlichi } \\
\text { Ferreira \& Domínguez, } 1992\end{array}$ & {$[\mathrm{SP}]$} & (Ferreira \& Domínguez 1992) \\
\hline $\begin{array}{l}\text { Hermanella (Guayakia) grandis } \\
\text { Domínguez \& Flowers, } 1989\end{array}$ & {$[\mathrm{PR}, \mathrm{SC}]$} & (Domínguez \& Flowers 1989) \\
\hline $\begin{array}{l}\text { Hermanella (Guayakia) } \\
\text { maculipennis (Ulmer, 1920) } \\
=\text { Thraulus maculipennis Ulmer, } \\
1920 \\
=\text { Traverella maculipennis (Ulmer, } \\
1920)\end{array}$ & {$[\mathrm{PR}, \mathrm{SC}]$} & (Ulmer 1920) \\
\hline $\begin{array}{l}\text { Hermanella (Hermanella) guttata } \\
\text { Domínguez \& Flowers, } 1989\end{array}$ & {$[\mathrm{PR}]$} & (Domínguez \& Flowers 1989) \\
\hline \multicolumn{3}{|l|}{ Hermanellopsis Demoulin, 1955} \\
\hline $\begin{array}{l}\text { Hermanellopsis arsia Savage \& } \\
\text { Peters, } 1983\end{array}$ & {$[\mathrm{AM}]$} & (Savage \& Peters 1983) \\
\hline
\end{tabular}




\begin{tabular}{|l|c|l|}
\hline \multicolumn{2}{|l|}{ Hylister Domínguez \& Flowers, 1989 } & (Domínguez \& Flowers 1989, Da- \\
\hline Hylister plaumanni Domínguez \& & [PR, SC; MG, RJ] & Silva 1997) \\
\hline Llowers, 1989 & [AM, RO, RR] & (Lopes 1999) \\
\hline Leentvaaria palpalis Demoulin, & & \\
\hline 1966 & & \\
\hline
\end{tabular}

Massartella Lestage, 1930

\begin{tabular}{|c|c|c|}
\hline $\begin{array}{l}\text { Massartella alegrettae Ulmer, } \\
1943\end{array}$ & [RS; RJ] & $\begin{array}{l}\text { (Ulmer 1943, Pescador \& Peters } \\
\text { 1990, Da-Silva 2002) }\end{array}$ \\
\hline $\begin{array}{l}\text { Massartella brieni (Lestage, 1924) } \\
=\text { Atalophlebia brieni Lestage, } \\
1924 \\
=\text { Atalophlebia axillata Navás, } \\
1934 \\
=\text { Massartella fruhstorferri Ulmer, } \\
1943\end{array}$ & [PR, RS; MG, RJ, SP] & $\begin{array}{l}\text { (Lestage 1924, Demoulin 1955, } \\
\text { Pescador \& Peters 1990, Da-Silva } \\
\text { \& Pereira 1993, Da-Silva 2002) }\end{array}$ \\
\hline
\end{tabular}

Microphlebia Savage \& Peters, 1983

\begin{tabular}{|l|c|l|}
\hline Microphlebia pallida Savage \& & [AM] & (Savage \& Peters 1983) \\
Peters, 1983 & [AM] & (Savage \& Peters 1983) \\
\hline Miroculis Edmunds, 1963 & [AM] & (Savage \& Peters 1983) \\
\hline Miroculis (Atroari) amazonicus & & \\
\hline Miroculis (Atroari) duckensis & &
\end{tabular}




\begin{tabular}{|c|c|c|}
\hline $\begin{array}{l}\text { Miroculis (Miroculis) brasiliaensis } \\
\text { Savage \& Peters, } 1983\end{array}$ & {$[\mathrm{DF}]$} & (Savage \& Peters 1983) \\
\hline $\begin{array}{l}\text { Miroculis (Miroculis) fittkaui } \\
\text { Savage \& Peters, } 1983\end{array}$ & {$[\mathrm{PA}]$} & (Savage \& Peters 1983) \\
\hline $\begin{array}{l}\text { Miroculis (Miroculis) marauiae } \\
\text { Savage \& Peters, } 1983\end{array}$ & {$[\mathrm{AM}]$} & (Savage \& Peters 1983) \\
\hline $\begin{array}{l}\text { Miroculis (Ommaethus) froehlichi } \\
\text { Savage \& Peters, } 1983\end{array}$ & {$[\mathrm{RJ}, \mathrm{SP}]$} & $\begin{array}{l}\text { (Savage \& Peters 1983, Da-Silva } \\
\text { 1997) }\end{array}$ \\
\hline $\begin{array}{l}\text { Miroculis (Ommaethus) mourei } \\
\text { Savage \& Peters, } 1983\end{array}$ & {$[\mathrm{PR}]$} & (Savage \& Peters 1983) \\
\hline $\begin{array}{l}\text { Miroculis (Yaruma) wandae } \\
\text { Savage \& Peters, } 1983\end{array}$ & {$[\mathrm{AM}]$} & (Savage \& Peters 1983) \\
\hline \multicolumn{3}{|c|}{ Needhamella Domínguez \& Flowers, 1989} \\
\hline $\begin{array}{l}\text { Needhamella ehrhardti (Ulmer, } \\
\text { 1920) } \\
=\text { Thraulus ehrhardti Ulmer, } 1920 \\
=\text { Traverella ehrhardti (Ulmer, } \\
\text { 1920) } \\
=\text { Hermanella sp.; Edmunds, } \\
\text { Jensen \& Berner, } 1976\end{array}$ & [PR, RS, SC; RJ; GO] & $\begin{array}{c}\text { (Ulmer 1920, Domínguez \& } \\
\text { Flowers 1989, Da-Silva 1997) }\end{array}$ \\
\hline \multicolumn{3}{|l|}{ Paramaka Savage \& Dominguez, 1992} \\
\hline $\begin{array}{l}\text { Paramaka convexa (Spieth, 1943) } \\
=\text { Thraulus convexus Spieth, } 1943 \\
=\text { Homothraulus convexus; Traver } \\
1960 \\
=\text { Hermanella sp.2 Demoulin, } 1966\end{array}$ & {$[\mathrm{PA}]$} & (Savage \& Domínguez 1992) \\
\hline Perissophlebiodes Savage, 1983 & & \\
\hline
\end{tabular}




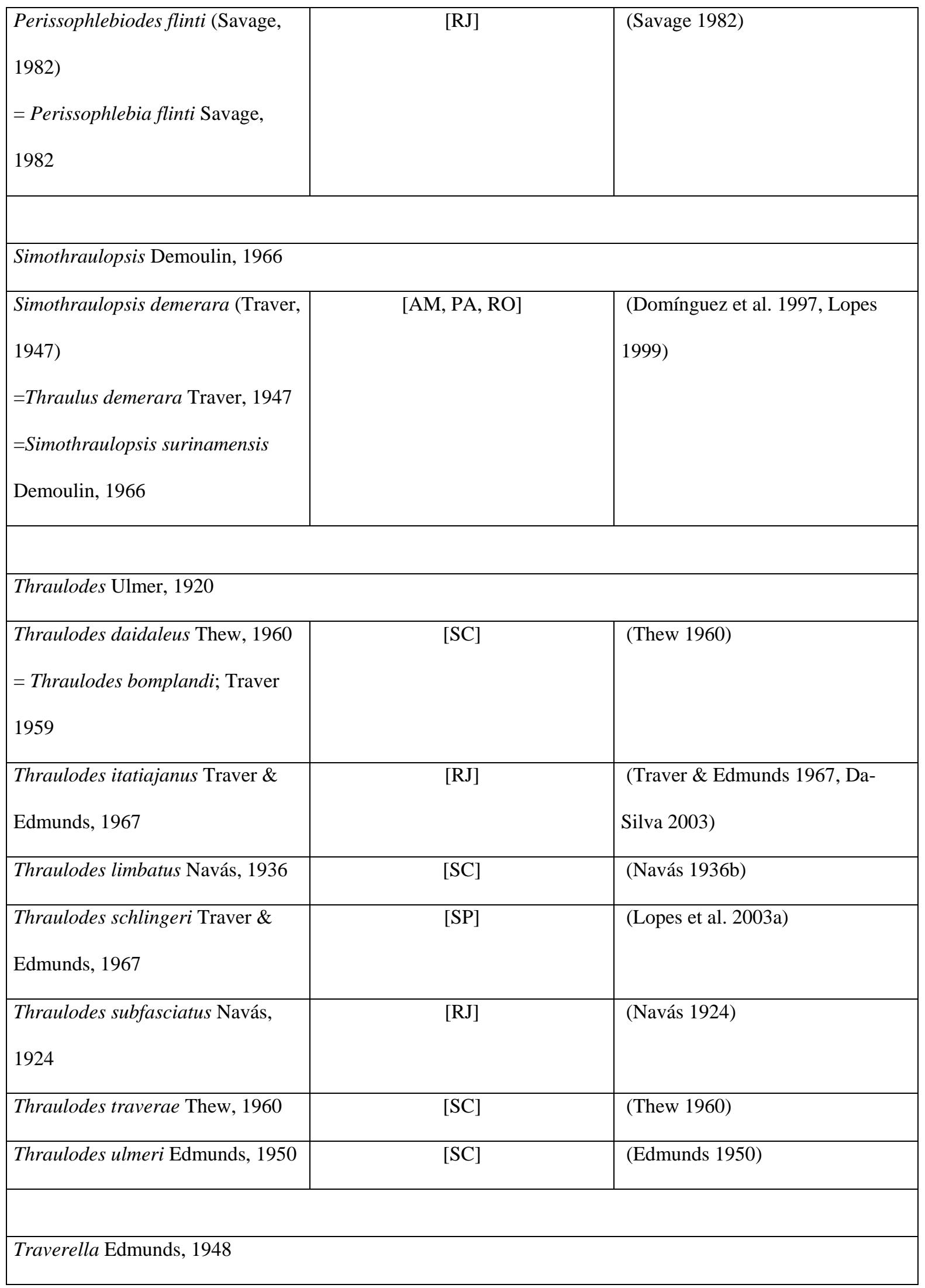




\begin{tabular}{|c|c|c|}
\hline $\begin{array}{l}\text { Traverella bradleyi (Needham \& } \\
\text { Murphy, 1924) } \\
\text { =Thraulus bradleyi Needham \& } \\
\text { Murphy, } 1924\end{array}$ & [REGIÃO CENTRO-OESTE] & (Needham \& Murphy 1924) \\
\hline Ulmeritoides Traver, 1959 & & \\
\hline $\begin{array}{l}\text { Ulmeritoides misionensis } \\
\text { Domíguez, } 1995\end{array}$ & {$[\mathrm{RO}]$} & $\begin{array}{l}\text { (Lopes 1999, Da-Silva \& Lopes } \\
\text { 2002) }\end{array}$ \\
\hline $\begin{array}{l}\text { Ulmeritoides patagiatus (Thew, } \\
\text { 1960) } \\
\text { =Ulmeritus patagiatus Thew, } 1960\end{array}$ & {$[\mathrm{SC}]$} & (Thew 1960) \\
\hline $\begin{array}{l}\text { Ulmeritoides oepa Lopes, Da-Silva } \\
\text { \& Py-Daniel, } 2003\end{array}$ & {$[\mathrm{RO}]$} & (Lopes et al. 2003b) \\
\hline $\begin{array}{l}\text { Ulmeritoides uruguayensis } \\
\text { (Traver, 1959) } \\
=\text { Ulmeritus (Ulmeritoides) } \\
\text { uruguayensis Traver, } 1959 \\
=\text { Ulmeritus uruguayensis (Traver, } \\
\text { 1959) } \\
=\text { Ulmeritus adustus Thew, } 1960 \\
=\text { Ulmeritus (Ulmeritoides) adustus } \\
\text { Thew, } 1960\end{array}$ & {$[\mathrm{SC}]$} & (Thew 1960) \\
\hline \multicolumn{3}{|l|}{ Ulmeritus Traver, 1956} \\
\hline $\begin{array}{l}\text { Ulmeritus balteatus Thew, } 1960 \\
=\text { Ulmeritus sp. Traver, } 1956 \\
=\text { Ulmeritus (Ulmeritus) balteatus } \\
\text { (Thew, 1960) }\end{array}$ & [SC] & (Thew 1960) \\
\hline
\end{tabular}




\begin{tabular}{|c|c|c|}
\hline $\begin{array}{l}\text { Ulmeritus saopaulensis (Traver, } \\
\text { 1946) } \\
=\text { Atalophlebioides sao-paulense } \\
\text { Traver, } 1946 \\
=\text { Ulmeritus (Ulmeritus) } \\
\text { saopaulensis (Traver, 1946) }\end{array}$ & {$[\mathrm{MG}, \mathrm{SP}]$} & $\begin{array}{l}\text { (Traver 1946, Da-Silva \& Pereira } \\
\text { 1992) }\end{array}$ \\
\hline \multicolumn{3}{|c|}{ FAMÍLIA MELANEMERELLIDAE (1 gênero, 1 espécie) } \\
\hline \multicolumn{3}{|l|}{ Melanemerella Ulmer, 1920} \\
\hline $\begin{array}{l}\text { Melanemerella brasiliana Ulmer, } \\
1920\end{array}$ & {$[\mathrm{ES}, \mathrm{SP}]$} & $\begin{array}{l}\text { (Ulmer 1920, Molineri \& } \\
\text { Domínguez 2003) }\end{array}$ \\
\hline
\end{tabular}

\section{FAMÍLIA OLIGONEURIIDAE (6 gêneros, 8 espécies)}

Fittkauneuria Pescador \& Edmunds, 1994

\begin{tabular}{|l|l|l|}
\hline Fittkauneuria adusta Pescador \& & [AM] & (Pescador \& Edmunds 1994) \\
Edmunds, 1994 & & \\
\hline
\end{tabular}

Homoeoneuria Eaton, 1881

\begin{tabular}{|l|c|l|}
\hline Homoeoneuria (Notochora) & [AM, BA] & (Pescador \& Peters 1980, Da-Silva \\
fittkaui Pescador \& Peters, 1980 & 1992) \\
\hline Lachlania Hagen, 1868 & [RJ] & (Da-Silva \& Pereira 1993) \\
\hline Lachlania boanovae Da-Silva \& & [RJ] & (Pereira 1987) \\
\hline Pereira, 1993 & [??] & (Pictet 1843) \\
\hline Oligoneuria Pictet, 1843 & & \\
\hline
\end{tabular}


Oligoneurioides Demoulin, 1955

\begin{tabular}{|l|l|l|}
\hline Oligoneurioides amazonicus & [AM] & (Demoulin 1955) \\
Demoulin, 1955 & & \\
\hline
\end{tabular}

Spaniophlebia Eaton, 1881

\begin{tabular}{|l|c|l|}
\hline Spaniophlebia assimilis Banks, & [REGIÃO NORTE] & (Banks 1913) \\
\hline Spaniophlebia trailae Eaton, 1881 & & \\
\hline
\end{tabular}

FAMÍLIA POLYMITARCYIDAE (3 gêneros, 26 espécies)

Asthenopus Eaton, 1871

Asthenopus curtus (Hagen, 1861)

[AM, PA]

(Hagen 1861, Demoulin 1955)

=Palingenia curta Hagen, 1861

=Campsurus curtus (Hagen, 1861)

=Campsurus amazonicus Hagen,

1888

=Asthenopus amazonicus (Hagen, 1888)

Asthenopus picteti (Hubbard, 1975)

=Palingenia albicans Pictet, 1843

=Campsurus albicans (Pictet, 1843)

=Asthenopus albicans (Pictet, 1843)

=Asthenopodes albicans (Pictet, 1843) 


\begin{tabular}{|c|c|c|}
\hline Campsurus Eaton, 1868 & & \\
\hline $\begin{array}{l}\text { Campsurus albicans (Percheron in } \\
\text { Guerin \& Percheron, 1838) } \\
=\text { Ephemera albicans Percheron, } \\
1838 \\
=\text { Palingenia albicans (Percheron, } \\
1838 \text { ) }\end{array}$ & [??] & (Guerin \& Percheron 1838) \\
\hline $\begin{array}{l}\text { Campsurus albifilum (Walker, } \\
1853 \text { ) } \\
=\text { Palingenia albifilum Walker, } \\
1853\end{array}$ & [RJ; PA] & (Walker 1853, Lestage 1923) \\
\hline \begin{tabular}{|l} 
Campsurus assimlis Traver, 1944 \\
\end{tabular} & [RS] & (Traver 1944) \\
\hline $\begin{array}{l}\text { Campsurus brasiliensis Traver, } \\
1944\end{array}$ & [RS] & (Traver 1944) \\
\hline $\begin{array}{l}\text { Campsurus burmeisteri Ulmer, } \\
1942\end{array}$ & [??] & (Ulmer 1921) \\
\hline $\begin{array}{l}\text { Campsurus claudus Needham \& } \\
\text { Murphy, } 1924\end{array}$ & [MG] & (Needham \& Murphy 1924) \\
\hline $\begin{array}{l}\text { Campsurus corumbanus Needham } \\
\text { \& Murphy, } 1924\end{array}$ & [MS; MG] & (Needham \& Murphy 1924) \\
\hline $\begin{array}{l}\text { Campsurus dorsalis (Burmeister, } \\
\text { 1839) } \\
=\text { Palingenia dorsalis Burmeister, } \\
1839 \\
=\text { Asthenopus dorsalis (Burmeister, } \\
1839)\end{array}$ & [RJ, SP; REGIÃO NORTE] & $\begin{array}{l}\text { (Banks 1913, Navás 1920c, } \\
\text { Lestage 1923) }\end{array}$ \\
\hline \begin{tabular}{|l} 
Campsurus duplicatus Spieth, \\
1943
\end{tabular} & [AM] & (Spieth 1943) \\
\hline Campsurus evanidus Neddham \& & {$[\mathrm{MG}]$} & (Needham \& Murphy 1924) \\
\hline
\end{tabular}




\begin{tabular}{|c|c|c|}
\hline Murphy, 1924 & & \\
\hline Campsurus indivisus Ulmer, 1942 & [??] & (Ulmer 1942) \\
\hline $\begin{array}{l}\text { Campsurus latipennis (Walker, } \\
\text { 1853) } \\
=\text { Palingenia latipennis Walker, } \\
1853\end{array}$ & [PA, REGIÃO NORTE] & (Walker 1853, Banks 1913) \\
\hline $\begin{array}{l}\text { Campsurus longicauda Navás, } \\
1931\end{array}$ & [SP] & (Navás 1931) \\
\hline $\begin{array}{l}\text { Campsurus lucidus Needham \& } \\
\text { Murphy, } 1924\end{array}$ & {$[\mathrm{SC}]$} & (Ulmer 1942) \\
\hline $\begin{array}{l}\text { Campsurus melanocephalus } \\
\text { Pereira \& Da-Silva, } 1991\end{array}$ & {$[\mathrm{RJ}]$} & (Pereira \& Da-Silva 1991) \\
\hline $\begin{array}{l}\text { Campsurus mutilus Needham \& } \\
\text { Murphy, } 1924\end{array}$ & [AM] & (Needham \& Murphy 1924) \\
\hline $\begin{array}{l}\text { Campsurus notatus Needham \& } \\
\text { Murphy, } 1924\end{array}$ & [MS; PA] & $\begin{array}{l}\text { (Needham \& Murphy 1924, } \\
\text { Demoulin 1955) }\end{array}$ \\
\hline $\begin{array}{l}\text { Campsurus quadridentatus Eaton, } \\
1871\end{array}$ & {$[\mathrm{PA}]$} & (Eaton 1871) \\
\hline $\begin{array}{l}\text { Campsurus segnis Needham \& } \\
\text { Murphy, } 1924\end{array}$ & {$[\mathrm{PA}]$} & (Needham \& Murphy 1924) \\
\hline $\begin{array}{l}\text { Campsurus striatus Needham \& } \\
\text { Murphy, } 1924\end{array}$ & [MS] & (Needham \& Murphy 1924) \\
\hline Campsurus truncatus Ulmer, 1920 & [ES] & (Ulmer 1920) \\
\hline Campsurus ulmeri Traver, 1950 & {$[\mathrm{SC}]$} & (Traver 1950) \\
\hline Campsurus zikani Navás, 1934 & {$[\mathrm{RJ}]$} & (Navás 1934) \\
\hline \multicolumn{3}{|l|}{ Tortopus Needham \& Murphy, 1924} \\
\hline Tortopus harrisi Traver, 1950 & [MS] & (Traver 1950) \\
\hline
\end{tabular}




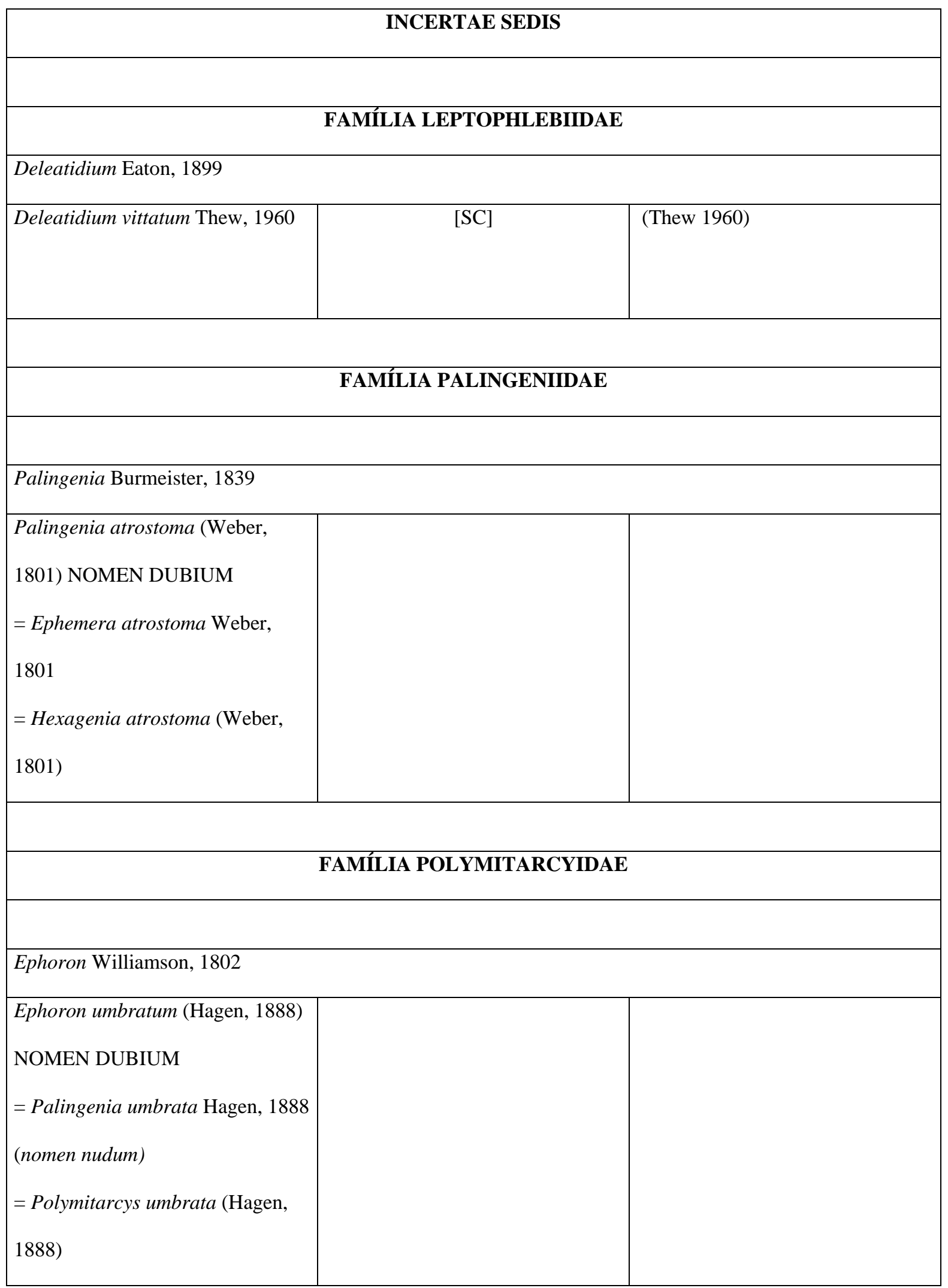


Tabela 2. Número de espécies de Ephemeroptera registrado para os estados brasileiros. PR, Paraná; RS, Rio Grande do Sul; SC, Santa Catarina. ES, Espírito Santo; MG, Minas Gerais; RJ, Rio de Janeiro; SP, São Paulo. DF, Distrito Federal; GO, Goiás; MS, Mato Grosso do Sul; MT, Mato Grosso. BA, Bahia. AC, Acre; AM, Amazonas; PA, Pará; RO, Rondônia; RR, Roraima. * indica que uma espécie está registrada para uma região, mas o estado no qual foi encontrada é desconhecido.

\begin{tabular}{|c|c|c|c|c|c|c|c|c|c|c|c|c|c|c|c|c|c|c|c|c|}
\hline \multirow[b]{2}{*}{ FAMILLIA } & \multicolumn{3}{|c|}{ REGIÃO SUL } & \multicolumn{5}{|c|}{ REGIÃO SUDESTE } & \multicolumn{5}{|c|}{ REGIÃO CENTRO-OESTE } & \multirow{2}{*}{\begin{tabular}{|c|} 
REGIÃO \\
NORDESTE
\end{tabular}} & \multicolumn{6}{|c|}{ REGIÃO NORTE } \\
\hline & PR & RS & SC & ES & MG & RJ & SP & * & DF & GO & MS & MT & * & & $A C$ & AM & PA & RO & RR & * \\
\hline Baetidae & 8 & 11 & 11 & 5 & 14 & 18 & 13 & 0 & 0 & 1 & 2 & 13 & 0 & 0 & 2 & 10 & 7 & 0 & 0 & 0 \\
\hline Caenidae & 0 & 0 & 0 & 0 & 0 & 2 & 1 & 0 & 0 & 0 & 0 & 2 & 2 & 0 & 0 & 6 & 4 & 0 & 0 & 1 \\
\hline Coryphoridae & 0 & 0 & 0 & 0 & 0 & 0 & 0 & 0 & 0 & 0 & 0 & 0 & 0 & 0 & 0 & 1 & 1 & 0 & 0 & 0 \\
\hline Ephemeridae & 1 & 0 & 0 & 0 & 0 & 0 & 1 & 0 & 0 & 0 & 0 & 0 & 0 & 0 & 0 & 0 & 1 & 0 & 0 & 0 \\
\hline Euthyplociidae & 0 & 2 & 0 & 1 & 1 & 1 & 2 & 0 & 0 & 0 & 0 & 0 & 0 & 0 & 0 & 1 & 1 & 0 & 0 & 0 \\
\hline Leptohyphidae & 3 & 4 & 7 & 0 & 0 & 4 & 0 & 1 & 0 & 1 & 0 & 1 & 0 & 0 & 0 & 1 & 1 & 0 & 0 & 0 \\
\hline Leptophlebiidae & 9 & 3 & 14 & 0 & 4 & 10 & 6 & 0 & 1 & 1 & 0 & 1 & 1 & 1 & 0 & 9 & 6 & 3 & 2 & 0 \\
\hline Melanemerellidae & 0 & 0 & 0 & 1 & 0 & 0 & 1 & 0 & 0 & 0 & 0 & 0 & 0 & 0 & 0 & 0 & 0 & 0 & 0 & 0 \\
\hline Oligoneuriidae & 0 & 0 & 0 & 0 & 0 & 2 & 0 & 0 & 0 & 0 & 0 & 0 & 0 & 1 & 0 & 4 & 0 & 0 & 0 & 1 \\
\hline Polymitarcyidae & 0 & 2 & 2 & 1 & 3 & 5 & 2 & 0 & 0 & 0 & 4 & 0 & 0 & 0 & 0 & 3 & 6 & 0 & 0 & 1 \\
\hline $\begin{array}{l}\mathrm{N} \text { total de } \\
\text { spp/estado }\end{array}$ & 21 & 22 & 34 & 8 & 22 & 42 & 26 & 1 & 1 & 3 & 6 & 17 & 3 & 2 & 2 & 35 & 27 & 3 & 2 & 3 \\
\hline
\end{tabular}

